Dynamic Factor Models for the Volatility Surface

Michel van der Wel, Sait R. Ozturk and Dick van Dijk

CREATES Research Paper 2015-13 


\title{
Dynamic Factor Models for the Volatility Surface
}

\author{
Michel van der Wel* Sait R. Ozturk Dick van Dijk \\ This version: January $30,2015^{\dagger}$
}

\begin{abstract}
The implied volatility surface is the collection of volatilities implied by option contracts for different strike prices and time-to-maturity. We study factor models to capture the dynamics of this three-dimensional implied volatility surface. Three model types are considered to examine desirable features for representing the surface and its dynamics: a general dynamic factor model, restricted factor models designed to capture the key features of the surface along the moneyness and maturity dimensions, and in-between spline-based methods. Key findings are that: (i) the restricted and spline-based models are both rejected against the general dynamic factor model, (ii) the factors driving the surface are highly persistent, (iii) for the restricted models option $\Delta$ is preferred over the more often used strike relative to spot price as measure for moneyness.
\end{abstract}

Keywords: Dynamic Factor Models, Implied Volatility Surface, Kalman filter, Maximum likelihood.

JEL: C32, C58, G13.

\footnotetext{
${ }^{*}$ Corresponding author, e-mail address: vanderwel@ese.eur.nl. Postal address: Erasmus University Rotterdam, Erasmus School of Economics, P.O.Box 1738, 3000 DR, Rotterdam, The Netherlands. Phone number: $+31(0) 10-4081316$.

†Van der Wel is from Erasmus University Rotterdam, CREATES, Tinbergen Institute and ERIM. Ozturk is from Erasmus University Rotterdam and the Tinbergen Institute. Van Dijk is from Erasmus University Rotterdam, Tinbergen Institute and ERIM. We appreciate the comments of participants at the 16th Annual Advances in Econometrics conference. Michel van der Wel is grateful to Netherlands Organisation for Scientific Research (NWO) for a Veni grant; and acknowledges support from CREATES - Center for Research in Econometric Analysis of Time Series (DNRF78), funded by the Danish National Research Foundation.
} 


\section{Introduction}

The value and pay-off of an option depends on the price of the underlying asset relative to the strike price (also called the moneyness) and the remaining time-to-maturity (or simply maturity). The maturity and strike are given in the option contract and for a given underlying asset typically a range of options with different maturities and strikes can be traded in financial markets. Because the prices of these different option contracts with the same underlying are difficult to interpret and compare, option prices often are converted into implied volatility. The implied volatility is obtained by backing out the volatility in such a way that the observed market price of the option contract matches the price implied by a certain pricing model, usually the Black and Scholes (1973) model or a binomial tree (introduced by Cox, Ross, and Rubinstein, 1979). The collection of implied volatilities across both the maturity and moneyness dimensions is referred to as the implied volatility surface. ${ }^{1}$ An extant literature, pioneered by Rubinstein (1994), shows that at any given point of time there are typical and common patterns for the implied volatility across the strike price (or moneyness more generally) and maturity dimensions. The pattern for given maturity across different strikes is often referred to as the volatility smile (because of its U-shape). The pattern for given moneyness across different maturities is referred to as the volatility term structure.

Since the pay-offs of option contracts for different strikes and maturities ultimately depend on the same underlying, a strong comovement in the different option prices is expected. As the implied volatility is a transformation of the prices, this feature carries over to the implied volatility surface. It is a natural idea to represent the comovement of different parts of the volatility surface in terms of common factors. However, there is no clear guidance in the literature on what type of factor model to use for this purpose. On one side of the spectrum, Fengler, Härdle, and Mammen (2007) suggest a flexible semiparametric factor model. On the other side of the spectrum, Christoffersen, Fournier, and Jacobs (2013) suggest a restricted factor model that decomposes the volatility surface into three factors representing

\footnotetext{
${ }^{1}$ The model used to obtain the volatility surface is not crucial. Important is that a (nonlinear) unique transformation of the prices is taken, to be able to compare options using a common unit of measurement. Another interpretation of the volatility surface is that outstanding options on the same underlying with the same maturity can be used to extract an implied risk-neutral distribution, which in turns implies a particular shape of the volatility surface. We are agnostic about the causes of the volatility surface and solely focus on the econometric modeling of the surface using factor modeling techniques.
} 
the implied volatility level, smile and term structure.

The contribution of this paper is to compare different factor model specifications for the implied volatility surface and examine desirable and undesirable features of such models. We use three different set-ups. First, we consider a general dynamic factor model (DFM) where only identification restrictions are imposed. Second, we examine restricted model specifications, where the factors are forced to represent comovement in the implied volatilities along the moneyness and maturity dimensions. Such a set-up is commonly used in the economics and finance literature, see, e.g., Dumas, Fleming, and Whaley (1998), Christoffersen, Fournier, and Jacobs (2013) and Christoffersen, Goyenko, Jacobs, and Karoui (2012). Third, we propose spline-based models that offer a flexible approach to capture the shape of the implied volatility surface. For this third set-up, we follow the smooth dynamic factor modeling approach of Jungbacker, Koopman, and van der Wel (2014). As in the second set-up, the factor loadings are structured in such a way that the corresponding factors represent smile and term structure effects, but the restrictions imposed on the loadings are much less strict. The spline-based models can be seen as a likelihood-based alternative of the semiparametric implementation using basis functions, as in Fengler, Härdle, and Mammen (2007) and Park, Mammen, Härdle, and Borak (2009). All the different DFM specifications are estimated with maximum likelihood adopting the framework of Jungbacker and Koopman (2014). Since both the restricted economic models and spline-based models are nested in the general dynamic factor model we use likelihood-ratio tests (LR-tests) to compare the models, besides comparing models based on information criteria.

We examine the merits of the different DFM set-ups in an empirical setting, using daily implied volatilities for European options on the S\&P500 index from 1999 through 2013. This is one of the most actively traded derivative securities, with contracts being available for a wide range of strike prices and maturities. We construct the implied volatility surface using six different groups of moneyness (measured by the $\Delta$ of the option ${ }^{2}$ ) and four different groups of time-to-maturity. On each day we find a contract nearest to the midpoint of each of the 24 moneyness-maturity pairs. We consider the balanced panel of the 24 daily selected contracts to capture the implied volatility surface.

\footnotetext{
${ }^{2}$ The $\Delta$ is defined as the sensitivity of the option price with respect to movements in the price of the underlying. The $\Delta$ is close to the implied probability that an option will end up in-the-money and is thus often used as measure for moneyness.
} 
Our results provide three key implications. First, the economic and spline-based models are both rejected against the general dynamic factor model; although the spline-based models perform much better than the restricted economic models. In all three model specifications, we find that the level of the volatility surface is the most important factor. The second and the third factors differ, however, and the preference for the general DFM suggests that the remaining comovement in the volatility surface does not correspond with the (economically plausible) smile and term structure effects as imposed by the restricted models. Second, for all estimated models the factors driving the surface are highly persistent. Third, to capture moneyness in the restricted economic models the option $\Delta$ performs better than the strike price relative to the spot price. This is an important implication, as many of the existing models use the strike relative to the spot price rather than $\Delta$. An explanation for this finding is that, unlike $\Delta$, the strike relative to the spot price does not take into account that the likelihood of the option being in-the-money at expiration depends on the (current) volatility of the stock and remaining time-to-maturity, as Bollen and Whaley (2004) point out.

We consider four extensions to our baseline model set-up. First, an alternative surface construction strategy is used based on moneyness measured by strike relative to spot price. Even when the data are constructed based on this measure, the models favor using $\Delta$ for moneyness. Second, while our main analysis is based on 3 -factor models we also examine higher-dimensional restricted models. We consider all models of Dumas, Fleming, and Whaley (1998), which have up to six factors. Only two of the 6-factor models provide a likelihood that is better to the general 3-factor DFM. Third, we consider alternatives for the factor dynamics and report similar results when random walks are taken for the factors. Fourth, the importance of the crisis period is examined by taking a log-transformation of the data and considering a sub-sample that omits the crisis period. While some factors are less persistent, the factor structure is also strong outside of the crisis period and when using $\operatorname{logs}$.

We contribute to two strands of literature. First, our approach contributes to the dynamic factor modeling literature by studying a surface. Many applications of factor models are 'two-dimensional' in nature and can be categorized as to whether the data can be logically structured in a particular way. Popular data sets as provided by Stock and Watson 
(2002) and related studies are a collection of macroeconomic variables where no immediate logical or natural ordering of the variables exists. However, in other cases such as Treasury yields, as in Jungbacker, Koopman, and van der Wel (2014), a natural ordering exists as the 3-month bond logically comes before the 6-month bond. Intermediate data sets are somewhat organized, such as housing prices as studied in Mönch and Ng (2011). We offer an approach to deal with organized three-dimensional data, by stacking the surface to get back to the case of two-dimensional data but benefiting from the resulting block-structure in the factor models.

Second, we contribute to the literature on the modeling of volatility surface. To the best of our knowledge we are the first to model the surface using a likelihood-based general dynamic factor approach. Alternative statistical implementations of factor models for the surface include Skiadopoulos, Hodges, and Clewlow (1999), Cont and da Fonseca (2002) and Fengler, Härdle, and Villa (2003). The restricted setting based on Dumas, Fleming, and Whaley (1998) has been extended by Gonçalves and Guidolin (2006) to include factor dynamics. The analysis is however done in a two-step framework, where the factors are first obtained by OLS and then modeled using vector-autoregressions. We provide an efficient approach to estimate the factors and their dynamics in one step. The closest paper to the spline-based set-up is Bedendo and Hodges (2009), who decompose the volatility smile using cubic polynomials and treat the knot values of these polynomials as factors. This can be seen as a restricted version of our approach and as the polynomials are based on moneyness it is fairly close to the restricted economic setting. Moreover we provide a unified framework for the surface, whereas the surface extension in their work is a separate treatment of the smile for different maturity groups.

The rest of the paper is organized as follows. Section 2 describes the data used in the analysis, summary statistics and a preliminary analysis of the data based on principal component analysis. Section 3 details the three different set-ups of the dynamic factor model. Section 4 provides the main estimation results. Section 5 discusses the outcomes of various robustness checks and extensions of the main modeling approach. Section 6 concludes. 


\section{Volatility Surface Data}

This section describes how we construct the implied volatility surface and provides a preliminary investigation of its characteristics. Knowledge of the key empirical features of the volatility surface provides useful input for the specification of the factor models as discussed in the next section. Section 2.1 discusses the data construction. Section 2.2 provides summary statistics and some insights into the possible usefulness of factor models based on principal component analysis.

\subsection{Constructing the Volatility Surface}

We use a daily dataset of European options on the S\&P500 index traded on the Chicago Board Options Exchange (CBOE). This is one of the most actively traded derivative securities. On an average day over a thousand option contracts are quoted. These vary along several dimensions: option type (call or put), expiry/maturity date, and strike price. The dataset, retrieved from OptionMetrics, consists of end-of-day values for all available option bid and ask quotes, as well as the corresponding time-to-maturity and strike price values. For each option contract, OptionMetrics calculates the implied volatility and other relevant characteristics, such as $\Delta$ and the strike price relative to spot price. Our sample period spans almost 15 years, from January 4, 1999, through August 30, 2013. Days on which the market is closed due to holidays or other reasons (such as the week following 9/11) are excluded, resulting in 3,688 daily observations.

The data are filtered to remove options that are inactive or may contain data errors. Our filtering procedures follow Barone-Adesi, Engle, and Mancini (2008). Specifically, we delete options (i) with a maturity longer than 360 days or shorter than 10 days, (ii) with an implied volatility above $70 \%,{ }^{3}$ (iii) with a price below $\$ 0.05$, or (iv) with missing values for either the implied volatility or $\Delta$. Moreover, we only consider out-of-the-money put and call options as these are more actively traded than in-the-money options. Because of the put-call parity considering out-of-the-money options is identical to studying in-the-money options or both types. Every in-the-money call (put) option can be matched to an out-of-the-money

\footnotetext{
${ }^{3}$ During the financial crisis there are a few days that volatility exceeds this level. We run a robustness analysis where we remove this second criteria and all results are similar.
} 
put (call) option, where the $\Delta$ of the call option is always one plus the $\Delta$ of the put option. For example, an in-the-money call option with a $\Delta$ of 0.75 should have the same implied volatility as an out-of-the-money put with a $\Delta$ of -0.25 .

We create daily implied volatility surfaces spanning the maturity and moneyness dimensions. We divide the data into four maturity groups, separated by maturities of 45, 90 and 180 days, and six moneyness groups of at-the-money, out-of-the-money, and deep out-ofthe-money options for both call and put options. Following Bollen and Whaley (2004), we define moneyness in terms of $\Delta$ because this also considers the volatility of the underlying asset, unlike the ratio of the strike price to the spot index price. We consider put options with $-0.125<\Delta<0$ as deep out-of-the-money (which we abbreviate with DOTM Put), with $-0.375<\Delta<-0.125$ as out-of-the-money (OTM Put), and with $-0.5<\Delta<-0.375$ as at-the-money (ATM Put). Similarly, we label call options with $0.375<\Delta<0.5$ as at-the-money (ATM Call), with $0.125<\Delta<0.375$ as out-of-the-money (OTM Call) and with $0<\Delta<0.125$ as deep out-of-the-money (DOTM Call). The combination of the four maturity and six moneyness groups provides 24 different groups, each containing a subset of all option contracts quoted on a day. For each maturity-moneyness group, we select the contract closest to the midpoint of both dimensions. ${ }^{4}$ On an average day there are 17 contracts in each group. Only if there is no option data within a group, we consider the closest contract across all groups. The approach to consider different groups in the large cross-section of data follows the literature, see, e.g., Bollen and Whaley (2004) and BaroneAdesi, Engle, and Mancini (2008). We consider a fairly large number of 24 groups, which is chosen to strike a balance between obtaining a balanced panel of similar contracts over the entire sample period and representing overall movements in the large cross-section of options. ${ }^{5}$

\subsection{Summary Statistics and Preliminary Analysis}

Figure 1 highlights some of the stylized facts about the implied volatility surface by displaying it for two specific days. Panel (A) shows the surface for June 4, 1999. The implied

\footnotetext{
${ }^{4}$ Closeness is defined by the summed squared distance for both $\Delta$ and maturity, where we put ten times more weight on $\Delta$ because the smaller values compared to maturity.

${ }^{5} \mathrm{An}$ alternative would be to consider the unbalanced set of all option contracts. While this poses no serious challenges for our modeling approach, we leave this for further research.
} 


\section{Figure 1: Volatility Surface on two days}

This figure shows the volatility surface on two days: June 4, 1999, and September 19, 2008. We show the implied volatility across four maturity groups and six moneyness groups. The maturity groups are 10-45 (group 1 in the figure), 45-90 (group 2), 90-180 (group 3) and 180-360 (group 4) days. The moneyness groups are $-0.125<\Delta<0$ (deep out-of-the-money put options, group 6 in the figure), $-0.375<\Delta<-0.125$ (out-of-the-money puts, group 5), $-0.5<\Delta<-0.375$ (at-the-money puts, group 4), $0.375<\Delta<0.5$ (atthe-money call options, group 3), $0.125<\Delta<0.375$ (out-of-the-money calls, group 2) and $0<\Delta<0.125$ (deep out-of-the-money calls, group 1). On each day we select the option that is closest to the middle of each maturity-moneyness group.

(A) June 4, 1999

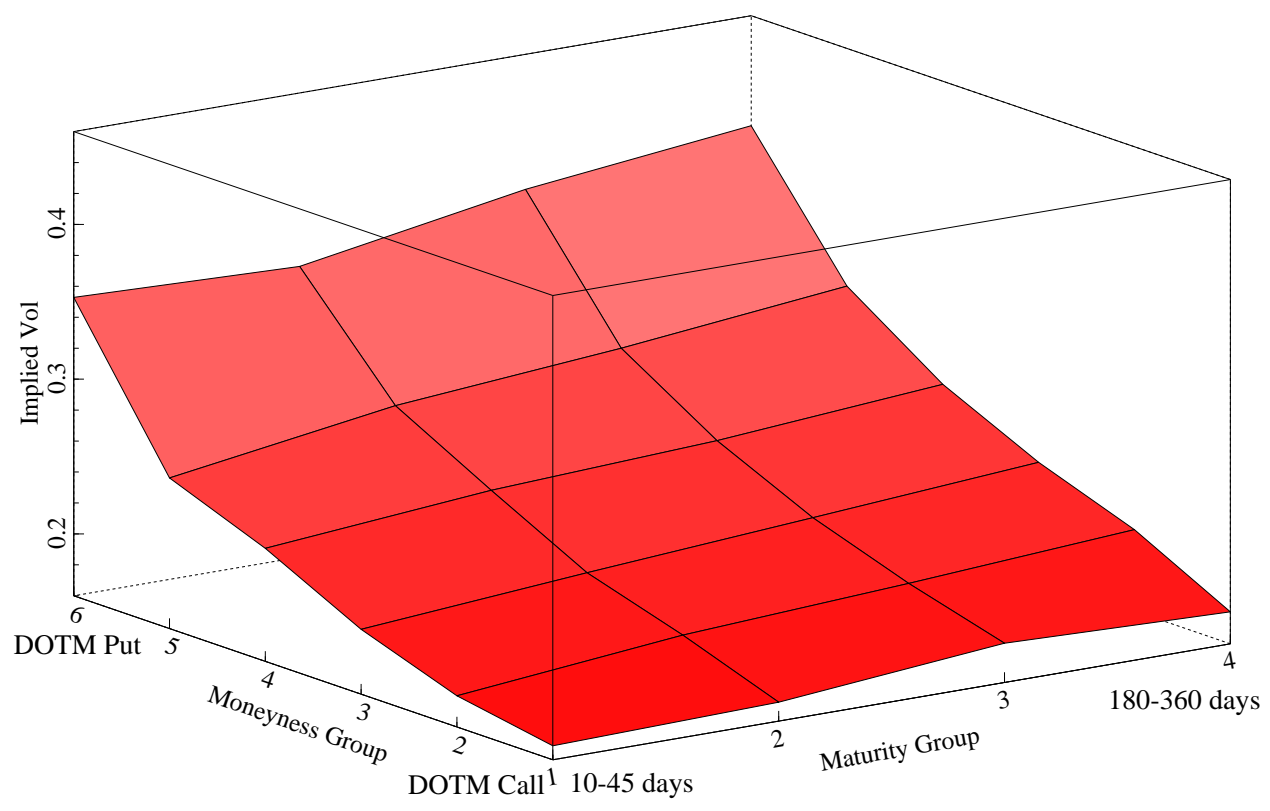

(B) September 19, 2008

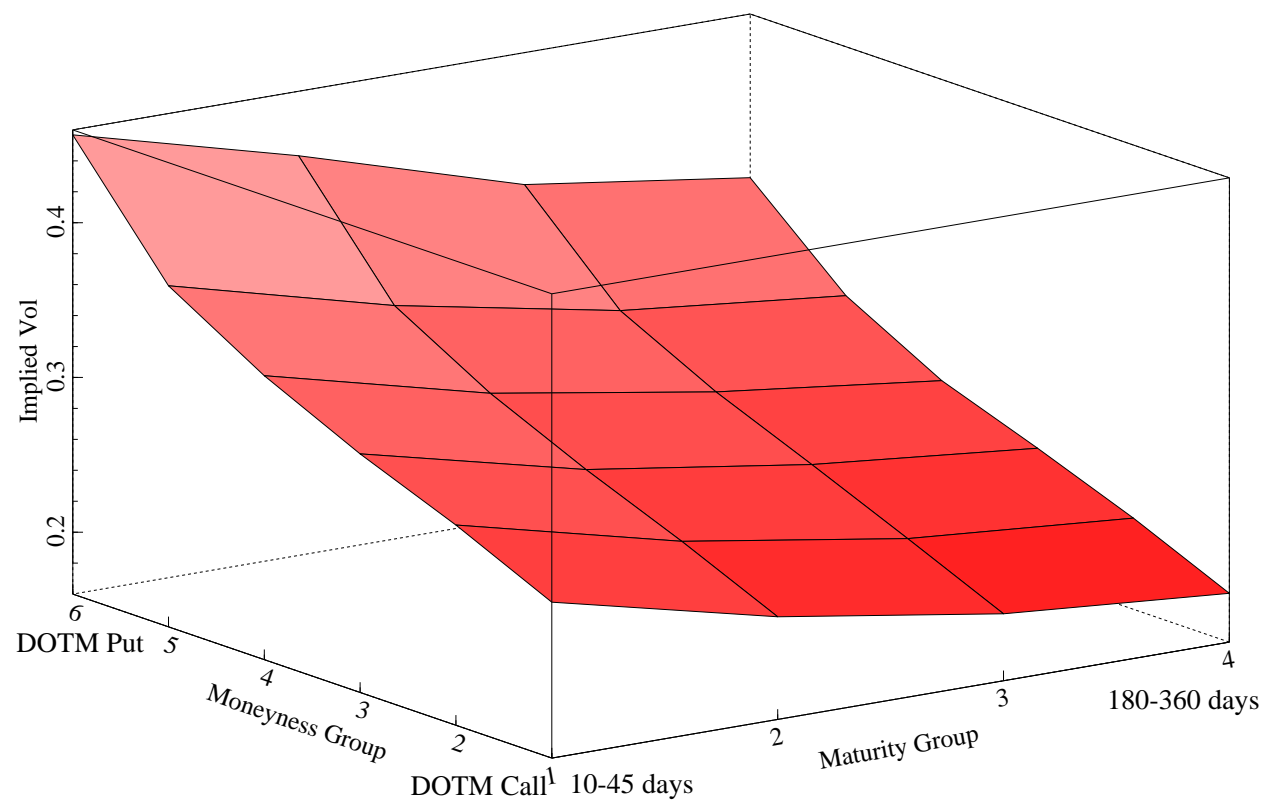


volatility slopes downward along the moneyness dimension for each of the four maturity groups. It is highest for deep out-of-the-money puts having low strike prices relative to the spot price and lowest for deep out-of-the-money calls with high strike prices relative to the spot price. This is a usual pattern and is commonly referred to as the volatility smile. ${ }^{6}$ The same pattern is indeed also found for September 19, 2008, a day during the height of the financial crisis, as shown in Panel (B). All implied volatilities are substantially higher compared to June 4, 1999, but the same smile is observed for all maturity groups. Interestingly, the implied volatility slopes (slightly) upward along the maturity dimension for June 4, 1999, while it slopes downward for September 19, 2008. A common explanation for this difference in the term structure is the mean-reversion of volatility: at relatively high levels of volatility the term structure slopes downward, while at low levels of volatility it slopes upward.

Table 1 provides summary statistics of the implied volatility surface. The table shows the time series mean and standard deviation of five variables for each of the 24 moneynessmaturity groups. For each group, statistics are reported for the mid-quote (in US dollars), the implied volatility, the option $\Delta$, the maturity (in days), and the strike relative to spot price $(K / S)$. The mid-quote increases with time-to-maturity and generally displays an inverse U-shape across the moneyness groups. The first feature reflects the options' time value, that is, options with longer maturity are traded at a higher price than short maturity options with comparable moneyness. The second feature is due to our definition of the moneyness groups and reflects the fact that options that are closer to being in-the-money are priced higher. The numbers for implied volatility confirm the observation based on Figure 1 that implied volatility slopes downward with moneyness. The average implied volatility for the DOTM Put group is almost the double of the average implied volatility for the DOTM Call group. The volatility term structure is fairly flat on average. The average option $\Delta$ is close to the midpoint of the relevant ranges defining the different moneyness groups and the same is the case for the average maturity. It is interesting to note that, whereas $\Delta$ is fairly constant across maturity groups, this is not the case for the strike price

\footnotetext{
${ }^{6}$ In stricter terms, a smile requires the implied volatility to slope upward again for high strike prices relative to the spot price. The observed pattern in Figure 1 is better described as a smirk due to the lack of such symmetry. In spite of this, we continue referring to the pattern as a smile due to the widespread usage of this term.
} 


\section{Table 1: Summary Statistics}

This table shows summary statistics for the option data. The table provides the mean and standard deviation ('Sd') over time for the mid-quote (in dollars), implied volatility ('Impl Vol'), option $\Delta$, maturity (in days), and strike relative to stock price $\left({ }^{\prime} K / S^{\prime}\right)$. We show these numbers across four maturity groups and six moneyness groups. The maturity groups are 10-45, 45-90, 90-180 and 180-360 days. The moneyness groups are $-0.125<\Delta<0$ (deep out-of-the-money put options, DOTM Put), $-0.375<\Delta<-0.125$ (out-of-themoney puts, OTM Put), $-0.5<\Delta<-0.375$ (at-the-money puts, ATM Put), $0.375<\Delta<0.5$ (at-themoney call options, ATM Call), $0.125<\Delta<0.375$ (out-of-the-money calls, OTM Call) and $0<\Delta<0.125$ (deep out-of-the-money calls, DOTM Call). On each day we find the option that is closest to the middle of each maturity-moneyness group. The numbers represent averages over time for the selected contracts in each group.

\begin{tabular}{|c|c|c|c|c|c|c|c|c|c|}
\hline \multicolumn{10}{|c|}{ Summary Statistics } \\
\hline & & \multicolumn{2}{|c|}{ 10-45 days } & \multicolumn{2}{|c|}{ 45-90 days } & \multicolumn{2}{|c|}{ 90-180 days } & \multicolumn{2}{|c|}{$180-360$ days } \\
\hline & & Mean & $\mathrm{Sd}$ & Mean & Sd & Mean & $\mathrm{Sd}$ & Mean & $\mathrm{Sd}$ \\
\hline \multirow[t]{5}{*}{ DOTM Put } & Mid-Quote & 2.63 & 2 & 4.47 & 2.15 & 6.48 & 2.23 & 9.99 & 3.42 \\
\hline & Impl Vol & 0.288 & 0.11 & 0.3 & 0.101 & 0.306 & 0.0925 & 0.308 & 0.0827 \\
\hline & $\Delta$ & -0.0627 & 0.0075 & -0.0631 & 0.00862 & -0.0627 & 0.00855 & -0.0643 & 0.0119 \\
\hline & Maturity & 27.9 & 10.7 & 67.8 & 9.45 & 133 & 23.3 & 269 & 26.2 \\
\hline & $K / S$ & 0.895 & 0.0422 & 0.834 & 0.0489 & 0.775 & 0.0578 & 0.705 & 0.0684 \\
\hline \multirow[t]{5}{*}{ OTM Put } & Mid-Quote & 10.9 & 4.72 & 18.6 & 6.18 & 27.6 & 8.22 & 42.1 & 11.9 \\
\hline & Impl Vol & 0.228 & 0.0957 & 0.234 & 0.086 & 0.239 & 0.0769 & 0.243 & 0.0674 \\
\hline & $\Delta$ & -0.25 & 0.0147 & -0.249 & 0.0154 & -0.25 & 0.0155 & -0.25 & 0.0178 \\
\hline & Maturity & 27.4 & 8.54 & 67.4 & 8.66 & 133 & 23.8 & 270 & 25.1 \\
\hline & $K / S$ & 0.964 & 0.0159 & 0.943 & 0.0199 & 0.923 & 0.0252 & 0.899 & 0.0326 \\
\hline \multirow[t]{5}{*}{ ATM Put } & Mid-Quote & 21.2 & 8.58 & 35.7 & 11.5 & 52.8 & 15.4 & 80.3 & 21.3 \\
\hline & Impl Vol & 0.204 & 0.0868 & 0.206 & 0.0764 & 0.209 & 0.0678 & 0.211 & 0.0587 \\
\hline & $\Delta$ & -0.437 & 0.0175 & -0.437 & 0.0185 & -0.437 & 0.0205 & -0.438 & 0.0162 \\
\hline & Maturity & 27.5 & 8.42 & 67.3 & 8.72 & 133 & 23 & 270 & 25.1 \\
\hline & $K / S$ & 0.994 & 0.00378 & 0.993 & 0.00643 & 0.993 & 0.0112 & 1 & 0.0195 \\
\hline \multirow[t]{5}{*}{ ATM Call } & Mid-Quote & 18.6 & 6.75 & 29.9 & 8.43 & 42.1 & 10.6 & 59.8 & 13.2 \\
\hline & Impl Vol & 0.191 & 0.0812 & 0.191 & 0.0698 & 0.193 & 0.0612 & 0.195 & 0.0528 \\
\hline & $\Delta$ & 0.437 & 0.0182 & 0.438 & 0.0201 & 0.438 & 0.0226 & 0.438 & 0.0188 \\
\hline & Maturity & 27.5 & 8.46 & 67.2 & 9.08 & 133 & 22.9 & 270 & 25.2 \\
\hline & $K / S$ & 1.01 & 0.00629 & 1.02 & 0.00984 & 1.03 & 0.0156 & 1.05 & 0.0247 \\
\hline \multirow[t]{5}{*}{ OTM Call } & Mid-Quote & 7.91 & 2.88 & 12.6 & 3.68 & 17.8 & 4.8 & 25.4 & 6.32 \\
\hline & Impl Vol & 0.177 & 0.075 & 0.174 & 0.063 & 0.175 & 0.0551 & 0.176 & 0.0473 \\
\hline & $\Delta$ & 0.25 & 0.0191 & 0.25 & 0.0219 & 0.25 & 0.0233 & 0.251 & 0.0215 \\
\hline & Maturity & 27.3 & 8.2 & 67.5 & 8.47 & 134 & 22.8 & 270 & 24.8 \\
\hline & $K / S$ & 1.03 & 0.0166 & 1.06 & 0.0235 & 1.08 & 0.0314 & 1.13 & 0.0429 \\
\hline \multirow[t]{5}{*}{ DOTM Call } & Mid-Quote & 1.32 & 0.495 & 2.08 & 0.707 & 2.91 & 1.08 & 4.43 & 1.78 \\
\hline & Impl Vol & 0.165 & 0.068 & 0.158 & 0.0561 & 0.157 & 0.0485 & 0.156 & 0.0412 \\
\hline & $\Delta$ & 0.0616 & 0.00906 & 0.0617 & 0.0108 & 0.0617 & 0.013 & 0.0656 & 0.0168 \\
\hline & Maturity & 27.3 & 8.2 & 67.3 & 8.74 & 133 & 23.1 & 267 & 28.9 \\
\hline & $K / S$ & 1.07 & 0.0336 & 1.11 & 0.0451 & 1.17 & 0.0591 & 1.25 & 0.078 \\
\hline
\end{tabular}


relative to spot price, illustrating that moneyness is measured differently by both variables.

Figures 2 and 3 provide time series plots of the volatility surface. Panel (A) of Figure 2 plots the average implied volatility across the 24 groups together with the VIX. Particularly the high volatility of the financial crisis during the second half of 2008 stands out, but also the increased uncertainty following 9/11, the second Gulf War in 2002-2003, the European sovereign debt crisis in 2010, and the debt-ceiling crisis of 2011. Panel (B) of the figure plots the time series of implied volatility for all 24 moneyness-maturity groups. We observe a strong comovement across the groups. During times of high volatility the dispersion across the groups is larger than the dispersion during times of low volatility. This is borne out more clearly in Figure 3, plotting the slope of the volatility smile (Panel $(\mathrm{A}))$ and the term structure (Panel (B)). The slope of the volatility smile in each maturity group is defined as the implied volatility of the deep out-of-the-money put options minus the implied volatility of the deep out-of-the-money calls. The slope of the volatility term structure is defined as the implied volatility of the longest maturity group minus the implied volatility of the shortest maturity group. Panel (A) shows that for all days in the sample the slope of the smile is positive for each of the maturity groups. Its magnitude varies considerably though, between 0.05 and 0.30, approximately. The smile is larger when the level of volatility is higher and is strongest during and following the financial crisis. The time series of the slope of the term structure in Panel (B) indicates the term structure can be both negative and positive. Consistent with the mean-reversion interpretation, during times of high volatility the term structure slopes downward, while it tends to slope upward during times of low volatility. Similar to the pattern of the average implied volatility, both the smile and term structure slopes show strong persistence.

To investigate the degree of comovement and persistence in the implied volatilities further, we analyze cross-correlations between the moneyness-maturity groups and (partial) autocorrelations within each group. Table A1 in the web appendix shows cross-correlations between the different moneyness groups for the shortest (10-45 days) and longest (180-360 days) maturity categories. Without exception, the cross-correlations are very high: Within a maturity category they all exceed 0.9 , but even across maturity categories they do not fall below 0.8. Also, cross-correlations decline as the difference in moneyness between the groups becomes larger. Table A2 in the web appendix reports (partial) autocorrelations 
Figure 2: Volatility Time Series

This figure shows the time series of implied volatility. Panel (A) plots the average implied volatility across the maturity-moneyness groups, together with the VIX. Panel (B) plots the time series for each of the 24 maturity-moneyness groups.

(A) Average Implied Volatility

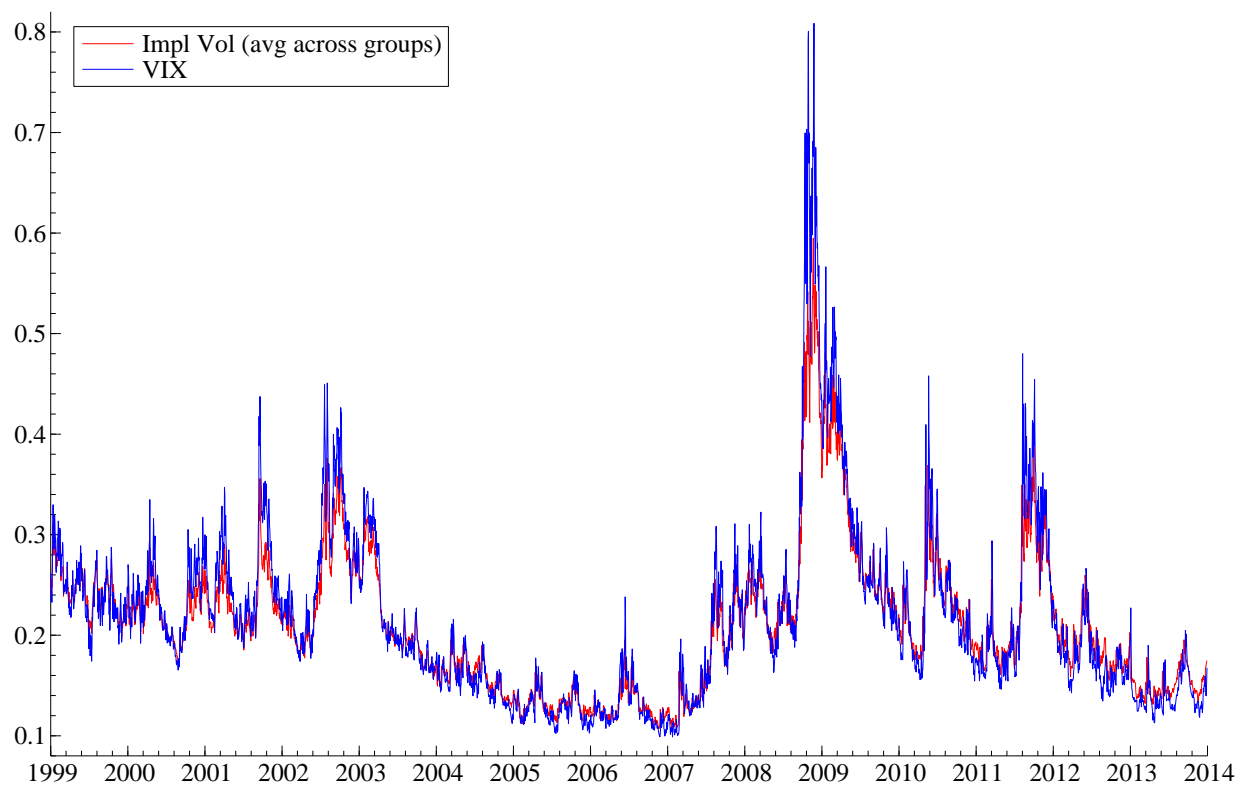

(B) Implied Volatility across Groups

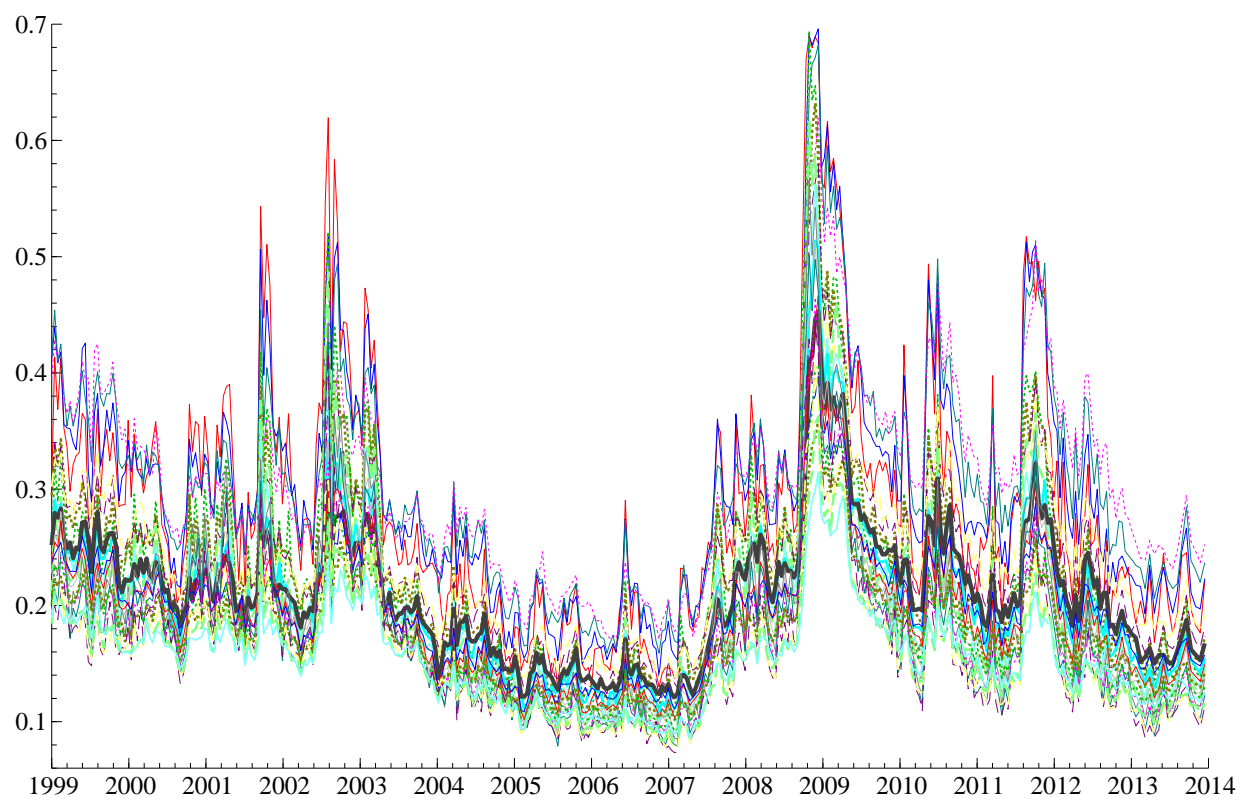




\section{Figure 3: Slope of the Volatility Smile and Term Structure}

This figure shows the time series of the implied volatility smile and term structure. Panel (A) plots the slope of the implied volatility smile. For each of the four maturity groups, we define the slope in that maturity group as the implied volatility of the deep out-of-the-money put minus the implied volatility of the deep out-of-the-money call. This is the implied volatility of the option with the lowest strike relative to stock price minus the implied volatility of the highest strike relative to the stock price. Panel (B) plots the slope of the volatility term structure. For each of the six moneyness groups, we define the slope as the implied volatility of the longest maturity minus the shortest maturity.

\section{(A) Slope of Volatility Smile}

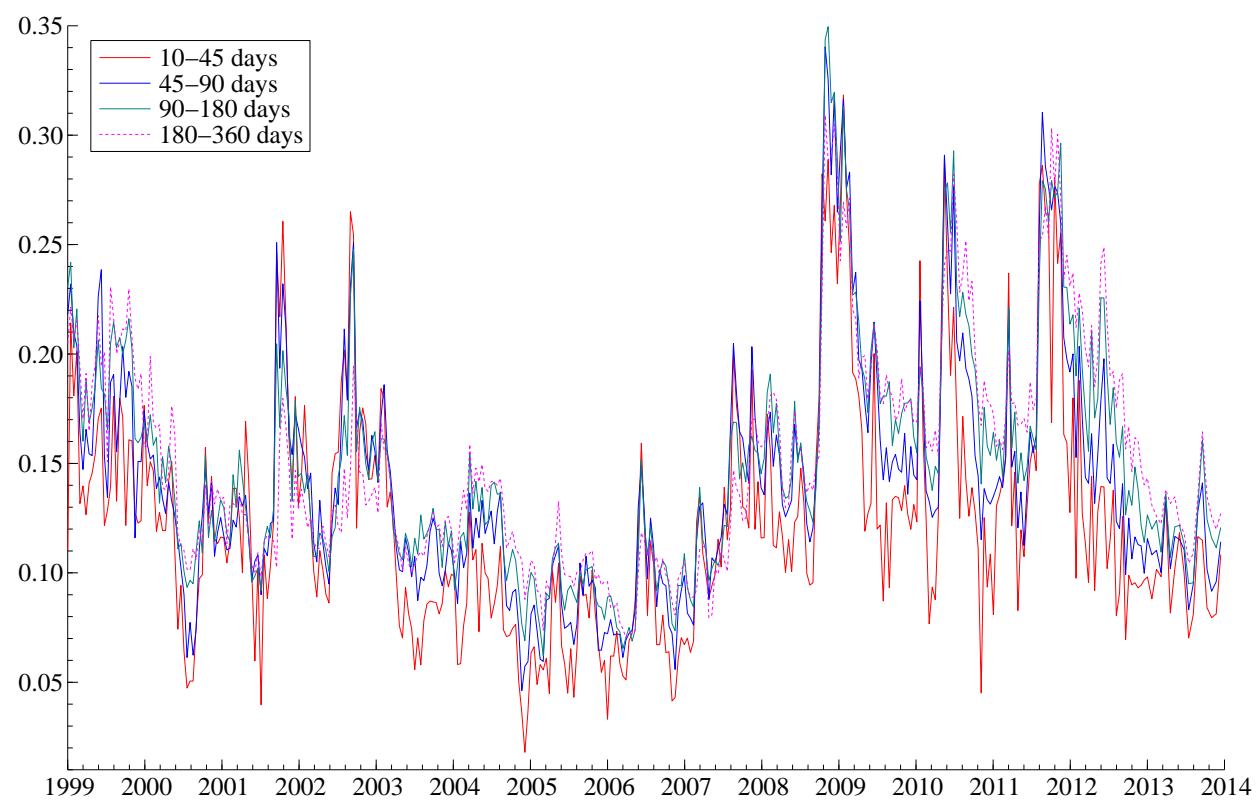

(B) Slope of Volatility Term Structure

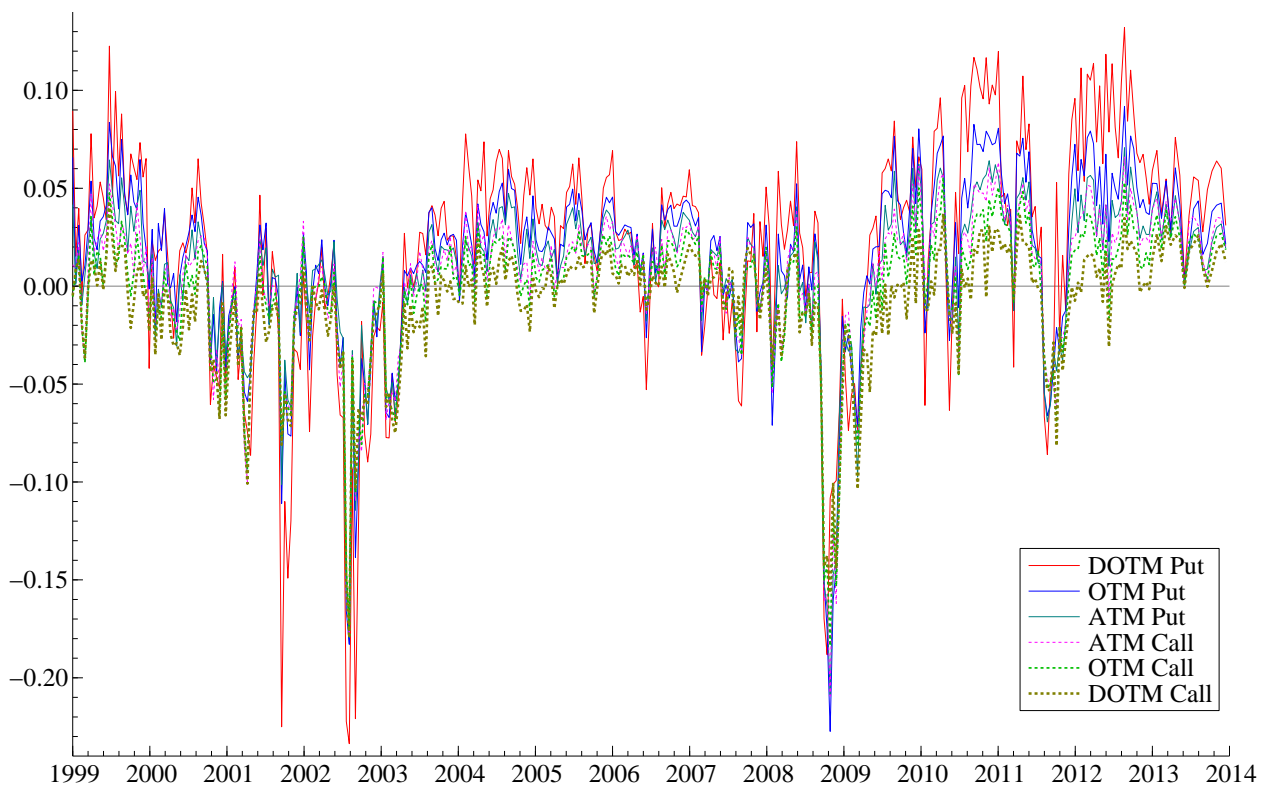


at various lags for a selection of implied volatilities, for the slope of the volatility smile at different maturities and for the slope of the term structure at different moneyness levels. We observe strong autocorrelations in each case, particularly for contracts with longer maturities. At the $20^{\text {th }} \mathrm{lag}$, the autocorrelation of the implied volatility stays above 0.8 for all contracts, while those of the slopes of the volatility smile and the term structure remain above 0.6 in all cases. For implied volatilities, the partial autocorrelations with the first lag are around 0.98 (they are by definition equal to the autocorrelations with the first lag) and they drop dramatically to the 0.1-0.25 range for the second lag. The two slope variables follow a similar shape in their partial autocorrelations.

The strong cross-correlations of the implied volatility across the moneyness-maturity groups suggests that it might be useful to employ common factors to describe the features of the implied volatility surface. To examine this further we run a principal component analysis. Table A3 in the web appendix provides the percentage of variation explained by the first 10 of the 24 principal components and the (partial) autocorrelation structure of the first three principal components. The bulk of the variation in the implied volatilities is captured by the first three principal components. Combined, they explain nearly $99 \%$ of the variation in the panel of 24 series. Figure A1 in the web appendix shows the time series of the first three principal components. The first principal component corresponds to the level of the implied volatility panel. The second and third component cannot immediately be linked to the volatility smile and term structure though. The principal component analysis confirms the presence of a strong factor structure in the implied volatility surface and motivates our set-up of dynamic factor modeling.

\section{Models for the Volatility Surface}

We now turn to the dynamic factor models for the implied volatility surface. We propose three different set-ups. Section 3.1 describes a general dynamic factor model, which nests all subsequent specifications. Section 3.2 discusses a restricted model recently proposed by Christoffersen, Fournier, and Jacobs (2013). This set-up explicitly takes the structure of the implied volatility surface data into account. Specifically, in addition to a factor representing the level of the surface, the model is forced to contain factors representing the smile and 
the (slope of the) term structure. This is achieved by setting the factor loadings equal to the moneyness and maturity of the corresponding option contract. Finally, Section 3.3 introduces related but more flexible specifications using splines to set the factor loadings along the moneyness and maturity dimensions.

\subsection{General Dynamic Factor Model}

The observation vector in our factor models consists of the vectorized volatility surface. Stacking the vector of implied volatilities for the $T$ different maturities for each of the $M$ moneyness groups results in the $(T M \times 1)$ vector of observations

$$
y_{t}=\left(\begin{array}{c}
I V_{\tau_{1}, m_{1}, t} \\
\vdots \\
I V_{\tau_{T}, m_{1}, t} \\
I V_{\tau_{1}, m_{2}, t} \\
\vdots \\
\vdots \\
I V_{\tau_{T}, m_{M}, t}
\end{array}\right),
$$

where $I V_{\tau_{i}, m_{j}, t}$ denotes the implied volatility on day $t$ for an option with time-to-maturity $\tau_{i}$, with $i=1,2, \ldots, T$, and moneyness $m_{j}$, with $j=1,2, \ldots, M$. In our application, we have $T=4$ maturities $T$ and $M=6$ moneyness categories. It is useful to note that because of the seasonality in option expiry dates and the variation in the option $\Delta$ (as shown in Table 1 ), it is not possible to use option contracts with exactly the same moneyness and maturity on each day and across the moneyness and maturity groups. For notational convenience, we suppress here the time-subscripts on the maturity $\tau$ and moneyness $m$ as well as the moneyness subscripts for maturity and the maturity subscripts for moneyness.

We start with a general dynamic factor model, given by

$$
\begin{aligned}
y_{t} & =\Lambda f_{t}+\varepsilon_{t}, & & \varepsilon_{t} \sim N\left(0, \Sigma_{\varepsilon}\right), \\
f_{t+1} & =\mu+\Phi\left(f_{t}-\mu\right)+\eta_{t+1}, & & \eta_{t+1} \sim N\left(0, \Sigma_{\eta}\right),
\end{aligned}
$$

where $y_{t}$ is the observation vector as defined above, $f_{t}$ are the $r$ latent dynamic factors 
that are loaded onto the implied volatilities using the $(T M \times r)$ factor loading matrix $\Lambda$ and $\varepsilon_{t}$ is the $(T M \times 1)$ vector of measurement errors with covariance matrix $\Sigma_{\varepsilon}$. Following standard practice and in line with the results of our (partial) autocorrelation analysis, we adopt a vector autoregressive (VAR) model of order one for the factors, with intercept $\mu$, VAR-coefficient matrix $\Phi$ and factor innovations $\eta_{t+1}$, which are assumed to be normally distributed with covariance matrix $\Sigma_{\eta}$.

The model is estimated using likelihood-based methods along the lines of Jungbacker and Koopman (2014). Motivated by the principal component analysis in Section 2.2, we focus on models with $r=3$ factors. To enable estimation, we impose identification restrictions. One possibility is to restrict the top-square part of the loading matrix $\Lambda$ and set it equal to the identity matrix, following Geweke and Zhou (1996). We implement a slight variation of this identification motivated by the empirical analysis of the volatility surface. We impose restrictions on four rows of $\Lambda$, corresponding to the shortest and longest maturities in the third and fourth moneyness groups (ATM Put and ATM Call), by setting

$$
\left(\begin{array}{ccc}
\lambda_{2 T+1,1} & \lambda_{2 T+1,2} & \lambda_{2 T+1,3} \\
\lambda_{3 T, 1} & \lambda_{3 T, 2} & \lambda_{3 T, 3} \\
\lambda_{3 T+1,1} & \lambda_{3 T+1,2} & \lambda_{3 T+1,3} \\
\lambda_{4 T, 1} & \lambda_{4 T, 2} & \lambda_{4 T, 3}
\end{array}\right)=\left(\begin{array}{ccc}
1 & -1 & 1 \\
1 & 1 & 1 \\
1 & -1 & -1 \\
1 & 1 & -1
\end{array}\right) .
$$

The restrictions force the first latent factor to capture the level of implied volatility across the four selected groups, the second factor to capture the term structure for the two moneyness groups and the third factor to capture the smile for the two maturity groups. Furthermore, we impose diagonality on the covariance matrix $\Sigma_{\varepsilon}$ of the measurement errors, implying that all comovement of the implied volatilities is attributed to the latent factors. The drop of the partial autocorrelations after the first lag of the principal components as reported in Table A3 motivates our use of a VAR with one lag. The VAR parameters in $\mu$ and $\Phi$ are left unrestricted and the parameters in $\Sigma_{\eta}$ are estimated using an LDL-decomposition. We initialize the latent factors from a standard normal distribution. ${ }^{7}$

\footnotetext{
${ }^{7}$ We also implement variants of a diffuse initialization with large variance and an initialization from the implied steady-state of the VAR. Results are qualitatively similar, though optimization for the steady-state initialization was less stable due to the strong persistence in the data.
} 


\subsection{Restricted Economic Dynamic Factor Models}

Given the structure of the implied volatility surface, in the context of DFMs it is quite natural to think of the latent factors as components representing the moneyness and maturity dimensions of the surface (or, in other words, the volatility smile and (the slope of) the term structure). Following this intuitively plausible idea, we adopt the setting of Christoffersen, Fournier, and Jacobs (2013) who extract the implied volatility level, moneyness slope and maturity slope by regressing the implied volatility cross-section at a given time $t$ on the moneyness and the maturity:

$$
I V_{\tau_{i}, m_{j}, t}=l_{t}+\tau_{i} c_{t}+m_{j} s_{t}+\varepsilon_{i, j, t}
$$

with $l_{t}$ the implied volatility level, $c_{t}$ the implied volatility maturity curve (along the maturity dimension), and $s_{t}$ the implied volatility smile (along the moneyness dimension). This approach can easily be cast in the dynamic factor framework. To do so, we collect the level, curve and smile into the latent state vector to obtain $f_{t}=\left(l_{t}, c_{t}, s_{t}\right)^{\prime}$. This results in a special case of the DFM in (1) with restricted factor loading matrix

$$
\Lambda=\left(\begin{array}{ccc}
1 & \tau_{1} & m_{1} \\
\vdots & \vdots & \vdots \\
1 & \tau_{T} & m_{1} \\
1 & \tau_{1} & m_{2} \\
\vdots & \vdots & \vdots \\
\vdots & \vdots & \vdots \\
1 & \tau_{T} & m_{M}
\end{array}\right)
$$

This loading matrix is deterministic and contains no parameters that need to be estimated.

Also here we have suppressed variation over time and across moneyness and maturity groups of the maturity $\tau_{i}$ and moneyness $m_{j}$. Because this variation may be useful for capturing the shape of the volatility surface, we run two variants. First, we consider specification with time-varying loading matrix $\Lambda_{t}$ containing the actual time-to-maturity and moneyness of each contract in the 24 moneyness-maturity groups on day $t$. Second, we consider a specification with a constant loading matrix $\Lambda$, where we take the time-series 
average of the maturities and moneyness for each of the 24 groups.

We also consider two different definitions of moneyness $m_{j}$. First, following Christoffersen, Fournier, and Jacobs (2013) we define moneyness as the ratio of the option's strike price $K$ and the spot price $S$. Second, following the constructing of our volatility surface data, we take moneyness equal to the option $\Delta$. As discussed in Section 2.1, the latter takes more properties of the data into account for defining the relative likelihood of the option ending up in-the-money. We consider both variables for both the constant and time-varying loading case. ${ }^{8}$ In all four of these restricted cases, we de-mean the second and third column to let the first factor capture movements in the level of the volatility surface (for the time-varying cases the columns are de-meaned on a daily basis).

\subsection{Spline-based Dynamic Factor Models}

While the idea that the latent factors in a DFM should capture the key features of the volatility surface along the maturity and moneyness dimensions is intuitively plausible, the approach of Christoffersen, Fournier, and Jacobs (2013) discussed above is quite restrictive in terms of the specification of the factor loadings. The third and final set-up is a hybrid approach, aiming to combine the flexibility of the general DFM of Section 3.1 with the economically plausible factor interpretation of the restricted model in Section 3.2. We propose to specify the factor loadings using splines in order to capture the shape of the volatility surface along both the maturity and moneyness dimensions in a flexible manner. The splines we use follow Poirier (1976) and have been used before in a dynamic factor modelling framework by Jungbacker, Koopman, and van der Wel (2014).

In a first variant of this approach, we impose relatively little structure on the loadings, in the sense that we do not impose restrictions on the loadings along the maturity dimension across the different moneyness groups, and vice versa. This is achieved by considering four separate splines across the moneyness dimension (one spline for each maturity group) and six separate splines across the maturity dimension (one for each moneyness group). Written

\footnotetext{
${ }^{8}$ To keep $\Delta$ comparable for put and call options we consider $1+\Delta$ for puts. This follows from the put-call parity, that states that the $\Delta$ of a call option minus the $\Delta$ of a put option with the same strike and maturity should be one. The necessity of this simple transformation is apparent from Table 1, where it can be seen that average $\Delta$ jumps from -0.437 to +0.437 when going from at-the-money put options to at-the-money call options.
} 
in a manner similar to the restricted models from the previous section, we have

$$
I V_{\tau_{i}, m_{j}, t}=l_{t}+f^{j}\left(\tau_{i}\right) c_{t}+g^{i}\left(m_{j}\right) s_{t}+\varepsilon_{i, j, t}
$$

with $f^{j}\left(\tau_{i}\right)$ the spline for maturity $i$ in moneyness group $j$, and $g^{i}\left(m_{j}\right)$ the spline for moneyness group $j$ with maturity $i$. The maturity splines $f^{j}(\cdot)$ capture the shape of the volatility term structure for the different moneyness groups, while the moneyness splines $g^{i}(\cdot)$ capture the shape of the volatility smile for the different maturity groups. Also this set-up can be written in the dynamic factor model framework, by using the factor loading matrix

$$
\Lambda=\left(\begin{array}{ccc}
1 & f^{1}\left(\tau_{1}\right) & g^{1}\left(m_{1}\right) \\
\vdots & \vdots & \vdots \\
1 & f^{1}\left(\tau_{T}\right) & g^{T}\left(m_{1}\right) \\
1 & f^{2}\left(\tau_{1}\right) & g^{1}\left(m_{2}\right) \\
\vdots & \vdots & \vdots \\
\vdots & \vdots & \vdots \\
1 & f^{M}\left(\tau_{T}\right) & g^{T}\left(m_{M}\right)
\end{array}\right)
$$

For each of the splines, a number of knots has to be selected. Given that $M=6$ the moneyness splines consist of at most six knots. The maturity splines consist of at most four knots, because $T=4$. The parameters to be estimated in the loading matrix are the knot values for each of the splines. For comparability with the restricted models of the previous section, we restrict the average knot values for each spline to be equal to zero. Besides the comparability, an advantage of this setting is that it imposes enough restrictions for the model to be identified. Note that in the case of six and four knots the model is identical to the general dynamic factor model of Section 3.1, apart from the identification restriction.

In the second variant of this approach, we restrict the moneyness splines $g^{i}(\cdot)$ to be the same across all maturity groups $i=1, \ldots, T$ and the maturity splines $f^{j}(\cdot)$ to be the same across all moneyness groups $j=1, \ldots, M$. This leads to the restricted specification for the observed implied volatility given by

$$
I V_{\tau_{i}, m_{j}, t}=l_{t}+f\left(\tau_{i}\right) c_{t}+g\left(m_{j}\right) s_{t}+\varepsilon_{i, j, t},
$$


with $f\left(\tau_{i}\right)$ the spline value for maturity $i$ and $g^{i}\left(m_{j}\right)$ the spline value for moneyness group $j$. In this case the factor loading matrix is given by

$$
\Lambda=\left(\begin{array}{ccc}
1 & f\left(\tau_{1}\right) & g\left(m_{1}\right) \\
\vdots & \vdots & \vdots \\
1 & f\left(\tau_{T}\right) & g\left(m_{1}\right) \\
1 & f\left(\tau_{1}\right) & g\left(m_{2}\right) \\
\vdots & \vdots & \vdots \\
\vdots & \vdots & \vdots \\
1 & f\left(\tau_{T}\right) & g\left(m_{M}\right)
\end{array}\right)
$$

Also for this variant, a number of knots has to be selected for each spline and the coefficients to be estimated are called as the knot values. Similarly, we impose the restriction that the average of the knot values is equal to zero.

A special case of this second variant is the specification with $T=4$ knots for the maturity spline and $M=6$ knots for the moneyness spline. In this case the knot values are simply the levels of each of the elements of the spline and the model is a block-version of the general dynamic factor model with

$$
\Lambda=\left(\begin{array}{ccc}
1 & \lambda_{1,1} & \lambda_{2,1} \\
\vdots & \vdots & \vdots \\
1 & \lambda_{1, T} & \lambda_{2,1} \\
1 & \lambda_{1,1} & \lambda_{2,2} \\
\vdots & \vdots & \vdots \\
\vdots & \vdots & \vdots \\
1 & \lambda_{1, T} & \lambda_{2, M}
\end{array}\right)
$$

with $T+M$ parameters to be estimated in the loading matrix, which reduces to $T+M-2$ parameters after imposing the restriction that the averages of the second and third columns must be equal to zero. 


\section{Main Results}

Table 2 provides key statistics concerning the fit of the three different DFM set-ups, estimated using the full sample of daily S\&P500 implied volatility surfaces over the period January 4, 1999 - August 30, 2013. The baseline model is the general dynamic factor model of Section 3.1. With three factors, the model contains 102 parameters in total. The estimated log-likelihood is 302,145, which serves as a benchmark for comparison with the other models.

Table 2: Comparing Dynamic Factor Models

This table provides statistics concerning the fit of the three dynamic factor models, when estimated using the full sample of daily S\&P500 implied volatility surfaces over the period January 4, 1999 - August 30, 2013.. The first model is the general dynamic factor model ('General DFM'), where only identification restrictions have been imposed. The second set of models are the restricted factor models based on the economic literature ('Restr DFM'). We estimate four variants, depending on whether the loading matrix is constant ('Const') or time-varying ('TV') and whether strike relative to stock price (' $K / S^{\prime}$ ') or $\Delta$ is used for moneyness. The third set of models are spline-based ('Spline DFM'). We consider the block dynamic factor model with knots on all places ('Block DFM'), a model with one spline for the maturity and one spline for the moneyness dimensions ('Mat and Mon Splines') and a model where there are separate splines for all maturity and moneyness groups ('Separate Splines'). For each model, we provide the log-likelihood value ('Loglik'), the number of parameters ('\#Pars') and the Akaike Information Criterion ('AIC'). For the restricted economic and spline models, we also provide a likelihood-ratio test ('LR-test') relative to the general dynamic factor model.

\begin{tabular}{|c|c|c|c|c|c|c|}
\hline \multicolumn{7}{|c|}{ Comparison of Factor Models } \\
\hline Model and 1 & oneyness & Const/TV & Loglik & LR-test & \#Pars & AIC \\
\hline \multicolumn{3}{|c|}{ General DFM (only ident restr) } & 302132.8 & & 102 & -604061.6 \\
\hline \multirow[t]{4}{*}{ Restr DFM } & $K / S$ & Const & 257089.0 & 90087.6 & 42 & -514094.0 \\
\hline & & $\mathrm{TV}$ & 261552.8 & 81182.4 & 42 & -522999.2 \\
\hline & $\Delta$ & Const & 263989.1 & 76287.4 & 42 & -542237.8 \\
\hline & & $\mathrm{TV}$ & 271160.9 & 61943.8 & 42 & -542260.8 \\
\hline \multirow[t]{3}{*}{ Spline DFM } & Block D & & 291583.0 & 21099.6 & 50 & -583066.0 \\
\hline & Mat ans & Mon Splines & 287181.4 & 29902.8 & 47 & -574268.8 \\
\hline & Separat & Splines & 293206.4 & 17852.8 & 66 & -586280.8 \\
\hline
\end{tabular}

For the restricted DFM discussed in Section 3.2, we consider four variants, where moneyness is measured either by the strike price relative to the spot price or $\Delta$ and where the loading matrix is either constant or time-varying. For all four variants we find much lower log-likelihood values, ranging between 257,089 and 271,160. This makes the general DFM specification preferred by both an LR-test and the Akaike Information Criterion. ${ }^{9}$ These

\footnotetext{
${ }^{9}$ Besides the Akaike Information Criterion we also consider the Schwartz Information Criterion which penalizes the general dynamic factor model more heavily due to its larger number of parameters. In all cases identical conclusions are drawn based on this alternative information criterion.
} 
measures take into account that the restricted models have far less parameters, namely only 42 compared to 102 in the general DFM. Among the four restricted models, the time-varying loading matrix is preferred over the constant loading matrix for both moneyness measures. For both the time-varying and constant loading case, $\Delta$ is preferred as measure of moneyness. This is striking, as many of the existing models use the strike relative to the spot price rather than $\Delta$ to measure moneyness.

As discussed in Section 3.3, we consider three variants of the spline-based dynamic factor models. Firstly, the most flexible case contains a separate moneyness spline for each maturity group and a separate maturity spline for each moneyness group. Secondly, a more restrictive case has a single spline for moneyness and a single spline for maturity. And thirdly, the block DFM constitutes a special case obtained when the number of knots is equal to the number of elements in the case of a single spline for moneyness and a single spline for maturity. All three variants perform a lot better than the restricted models. For example, the log-likelihood of the block dynamic factor model is more than 20,000 points higher than the best restricted model even though the loading matrix is constant. For each of the splines, we select three knots for the maturity spline (out of maximum four) and four for the moneyness spline (out of maximum six). Due to the restriction that the average of the knot values is zero, two and three parameters have to be estimated for the splines to model the maturity and moneyness dimensions, respectively. The maturity and moneyness spline variant performs worse than the block dynamic factor model, even though the difference in the number of parameters is very small. The separate splines variant performs best. Out of all restricted and spline models, it comes closest to the general dynamic factor model. Nevertheless, it is still rejected by the LR-test and also not preferred by the Akaike Information Criterion. ${ }^{10}$

In the remainder of this section, we show some further estimation output for a selection of the models. We focus on one variant for each of the three model types. Besides the general dynamic factor model, we also consider the restricted model where $\Delta$ is used as

\footnotetext{
${ }^{10}$ As expected, the model fit improves as the number of knots increase. For example, when considering three knots for the maturity spline and five for the moneyness spline the likelihood for the model with one maturity and one moneyness spline increases from 287,181.4 to 291,485.0. The location of knots also matters. A variant with the same number of knots (three and four) but on different location provides a likelihood of $288,064.8$. We leave selection of the number of knots and the location of knots as a topic for further research.
} 
the measure of moneyness. For the spline-based models, we consider the most flexible case with separate splines for maturity (moneyness) across moneyness (maturity) groups. Our motivation for this selection is that these models provided the highest log-likelihood within each model class. For the restricted model, we consider the constant factor loading case, for comparability with the other models.

\section{Table 3: Factor Dynamics}

This table provides the estimates of the factor dynamics for three of the factor models we consider. We show the coefficients for the general dynamic factor model (Panel A), the restricted dynamic factor model with a constant loading matrix where moneyness is measured with $\Delta$ (Panel $\mathrm{B})$, and the spline dynamic factor model where there is a separate spline for each moneyness and maturity group (Panel C). For all three models, we plot the intercept $\mu$, VAR coefficient matrix $\Phi$ and the innovation variance $\Sigma_{\eta}$.

\begin{tabular}{|c|c|c|c|c|c|c|c|}
\hline \multicolumn{8}{|c|}{ Panel A: Factor Dynamics General DFM } \\
\hline & \multirow[b]{2}{*}{$\mu$} & \multicolumn{3}{|c|}{$\Phi$} & \multicolumn{3}{|c|}{$\Sigma_{\eta}\left(\times 10^{-4}\right)$} \\
\hline & & $f_{1, t-1}$ & $f_{2, t-1}$ & $f_{3, t-1}$ & $f_{1, t}$ & $f_{2, t}$ & $f_{3, t}$ \\
\hline$f_{1, t}$ & 0.196 & 0.995 & 0.028 & -0.005 & 1.059 & -0.453 & 0.051 \\
\hline$f_{2, t}$ & 0.003 & -0.002 & 0.971 & 0.056 & -0.453 & 0.212 & -0.022 \\
\hline$f_{3, t}$ & 0.008 & 0.001 & 0.001 & 0.967 & 0.051 & -0.022 & 0.005 \\
\hline
\end{tabular}

\begin{tabular}{|c|c|c|c|c|c|c|c|}
\hline \multicolumn{8}{|c|}{ Panel B: Factor Dynamics Restricted DFM } \\
\hline & \multirow[b]{2}{*}{$\mu$} & \multicolumn{3}{|c|}{$\Phi$} & \multicolumn{3}{|c|}{$\Sigma_{\eta}\left(\times 10^{-4}\right)$} \\
\hline & & $l_{t-1}$ & $c_{t-1}$ & $s_{t-1}$ & $l_{t}$ & $c_{t}$ & $s_{t}$ \\
\hline$l_{t}$ & 0.200 & 0.995 & 0.054 & 0.006 & 0.939 & -0.220 & 0.129 \\
\hline$c_{t}$ & 0.001 & -0.001 & 0.969 & 0.003 & -0.220 & 0.057 & -0.027 \\
\hline$s_{t}$ & 0.032 & 0.001 & 0.002 & 0.990 & 0.129 & -0.027 & 0.029 \\
\hline
\end{tabular}

\begin{tabular}{|c|c|c|c|c|c|c|c|}
\hline \multicolumn{8}{|c|}{ Panel C: Factor Dynamics Spline DFM } \\
\hline & \multirow[b]{2}{*}{$\mu$} & \multicolumn{3}{|c|}{$\Phi$} & \multicolumn{3}{|c|}{$\Sigma_{\eta}\left(\times 10^{-2}\right)$} \\
\hline & & $f_{1, t-1}$ & $f_{2, t-1}$ & $f_{3, t-1}$ & $f_{1, t}$ & $f_{2, t}$ & $f_{3, t}$ \\
\hline$f_{1, t}$ & 0.207 & 0.995 & 0.001 & 0.000 & 0.012 & -0.169 & 0.035 \\
\hline$f_{2, t}$ & 0.135 & -0.094 & 0.967 & 0.025 & -0.169 & 2.548 & -0.442 \\
\hline$f_{3, t}$ & 0.694 & 0.017 & 0.001 & 0.988 & 0.035 & -0.442 & 0.143 \\
\hline
\end{tabular}

Figure 4 presents the estimated latent factors for the three selected models. The first factor is very similar across the models and captures the overall level of the volatility surface. This is expected, because in all cases the second and third columns of the loading matrix are de-meaned. The second factor also seems fairly similar across the three model types, though the scaling varies. This variation is particularly the case for the spline-based model. Due to the identification restriction for the splines, the factor loading matrix has smaller entries in the second (and third) column and hence the factors have larger variation. The 
Figure 4: Estimated Factors

This figure shows the three dynamic factors for three of the factor models we consider. We show the smoothed factors for the general dynamic factor model (Panel A), the restricted dynamic factor model with a constant loading matrix where moneyness is measured with $\Delta$ (Panel B), and the spline dynamic factor model where there is a separate spline for each moneyness and maturity group (Panel $\mathrm{C}$ ). The red line represents the first factor, the blue line the second factor and the red line the third factor.

(A) General Dynamic Factor Model

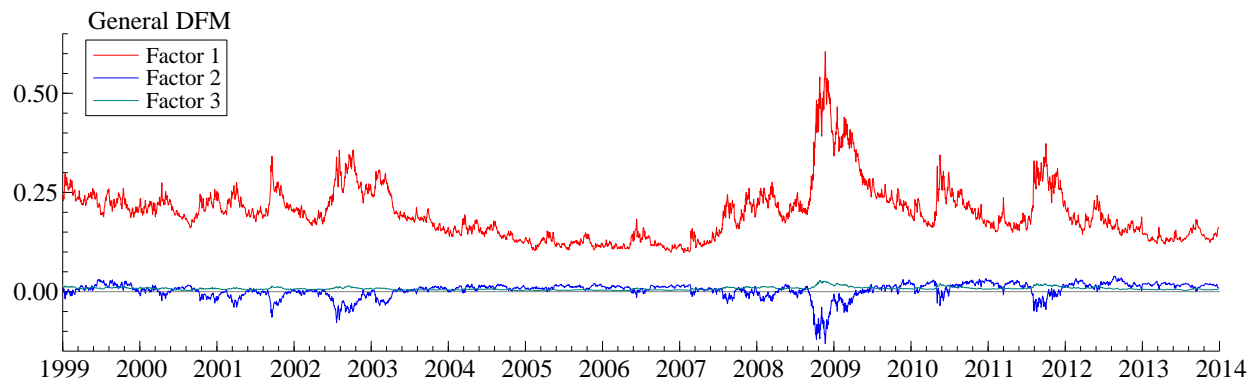

(B) Restricted Economic Dynamic Factor Model

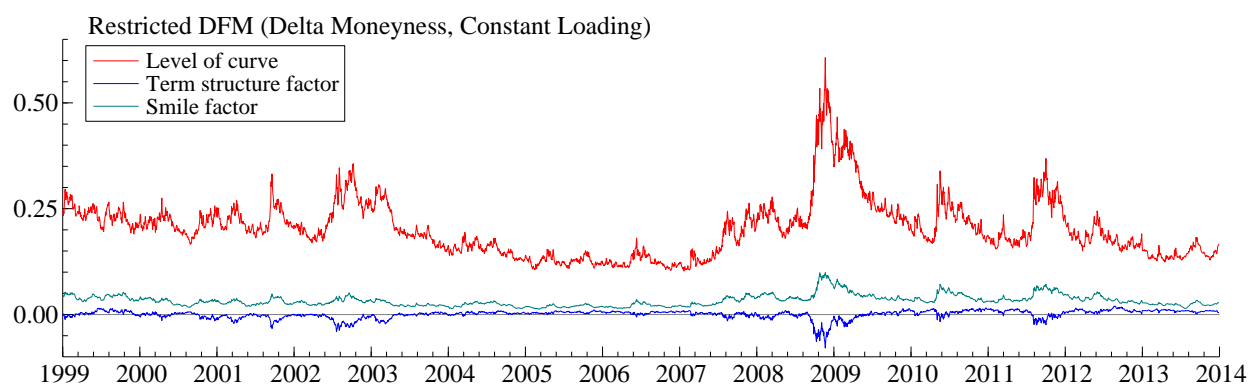

(C) Spline Dynamic Factor Model

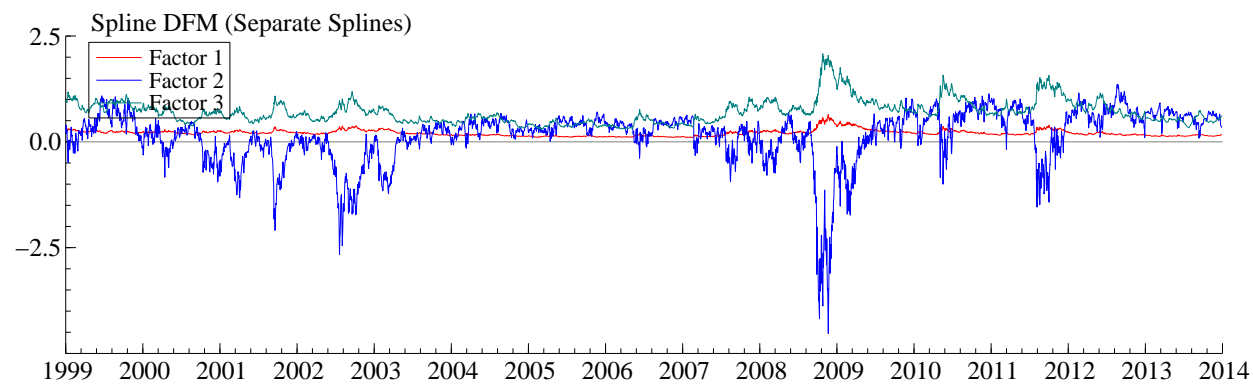


third factor differs even more across the models, particularly for the general dynamic factor model compared to the two restricted models.

Due to the different model structure and loading matrix the factors may seem different, but this might just be due to a rotation. To examine this possibility, we rotate the general dynamic factor model to each of the two other models. ${ }^{11}$ Panel A of Figure A2 in the web appendix confirms that the first and second factors of the general and restricted factor models are similar. However, the third factor remains different, even after rotation. From Panel B it is clear that the spline model is much closer to the general dynamic factor model, something which was already suggested by the log-likelihood values.

Table 3 documents the factor dynamics. All factors are very persistent, with diagonal elements very close to one. The off-diagonals are mostly zero. There is a strong correlation between the factor innovations for all models. For the general dynamic factor model, this correlation is -0.95 between the first and second factor, 0.72 between the first and third factor, and -0.70 between the second and third factor. ${ }^{12}$ The correlations are similar for the other models. The different scaling of the second and third factor for the spline-based model compared to the other models is also clear from the estimated covariance matrix. While the variance of the first factor is roughly in the same order of size as it is in the other models, the variance of the second and third factors are more than a 100 times higher.

Figure 5 provides further explanation for the different findings from the three models. Based on the factor loading matrix, for all the models an implied term structure is constructed for all six moneyness groups and an implied smile is constructed for all four maturity groups. The restricted models allow for very little variation in the type of term structure and smile. The unrestricted and spline-based factor models offer a lot more variation. This is a likely reason for the relatively poor performance of the restricted economic dynamic factor models. Also the difference in scaling between the spline-based model compared to the other two models is visible, explaining the larger variance for the second and third factors in the spline-based case.

Finally, Figure 6 provides some plots for the fit of the general dynamic factor model.

\footnotetext{
${ }^{11}$ This is done by regressing the columns of one loading matrix on the columns of the other loading matrix. The resulting square rotation matrix can be used to construct a best-fitting rotated loading matrix. By pre-multiplying the factors with the inverse of this rotation matrix the rotated factors are found.

${ }^{12}$ These correlations are based on the covariance matrix reported in Table 3.
} 


\section{Figure 5: Shape of Implied Volatility Term Structure and Smile}

This figures shows the volatility term structure and volatility smile that is implied by three of the factor models we consider. We show the term structure and smile for the general dynamic factor model (Panel A), the restricted dynamic factor model with a constant loading matrix where moneyness is measured with $\Delta$ (Panel B), and the spline dynamic factor model where there is a separate spline for each moneyness and maturity group (Panel C). In all panels the left figure depicts the volatility term structure and the right picture the volatility smile. The term structure and smile are implied by the factor loading matrix for each model.

(A) Term Structure and Smile - General Dynamic Factor Model
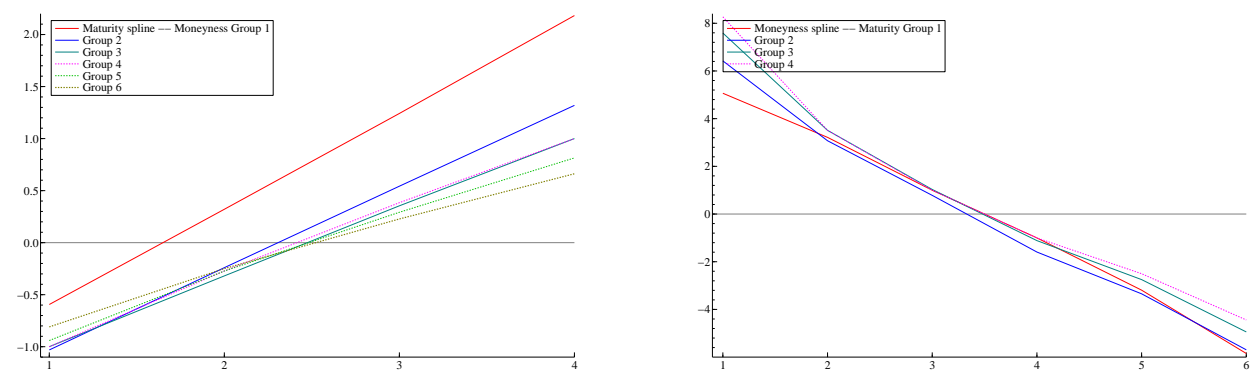

(B) Term Structure and Smile - Restricted Economic Dynamic Factor Model
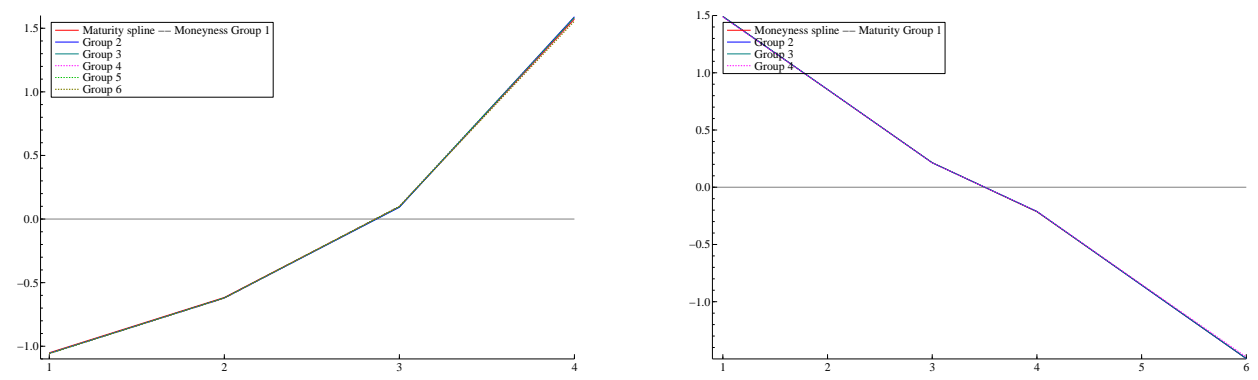

(C) Term Structure and Smile - Spline Dynamic Factor Model
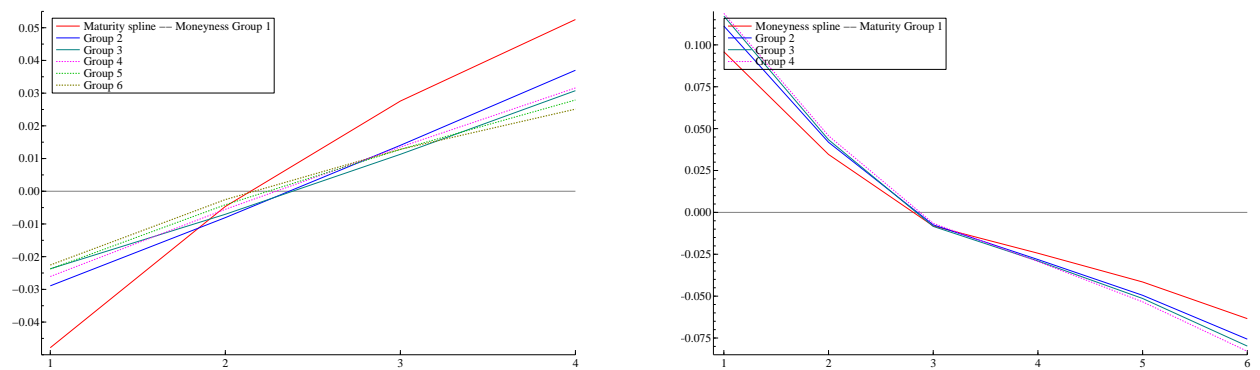


\section{Figure 6: Fit}

This figure documents the fit of the volatility surface based on the general dynamic factor model. We show the smoothed fitted values, which are obtained by pre-multiplying the smoothed factors with the estimated factor loading matrix. In the figure the red line is the actual implied volatility, the blue line the fitted and the green line the residual. We show six different maturity-moneyness combinations: the top plots depict two points in the middle of the surface and the bottom plots four outer points on the surface.

\section{Fit for General Dynamic Factor Model}
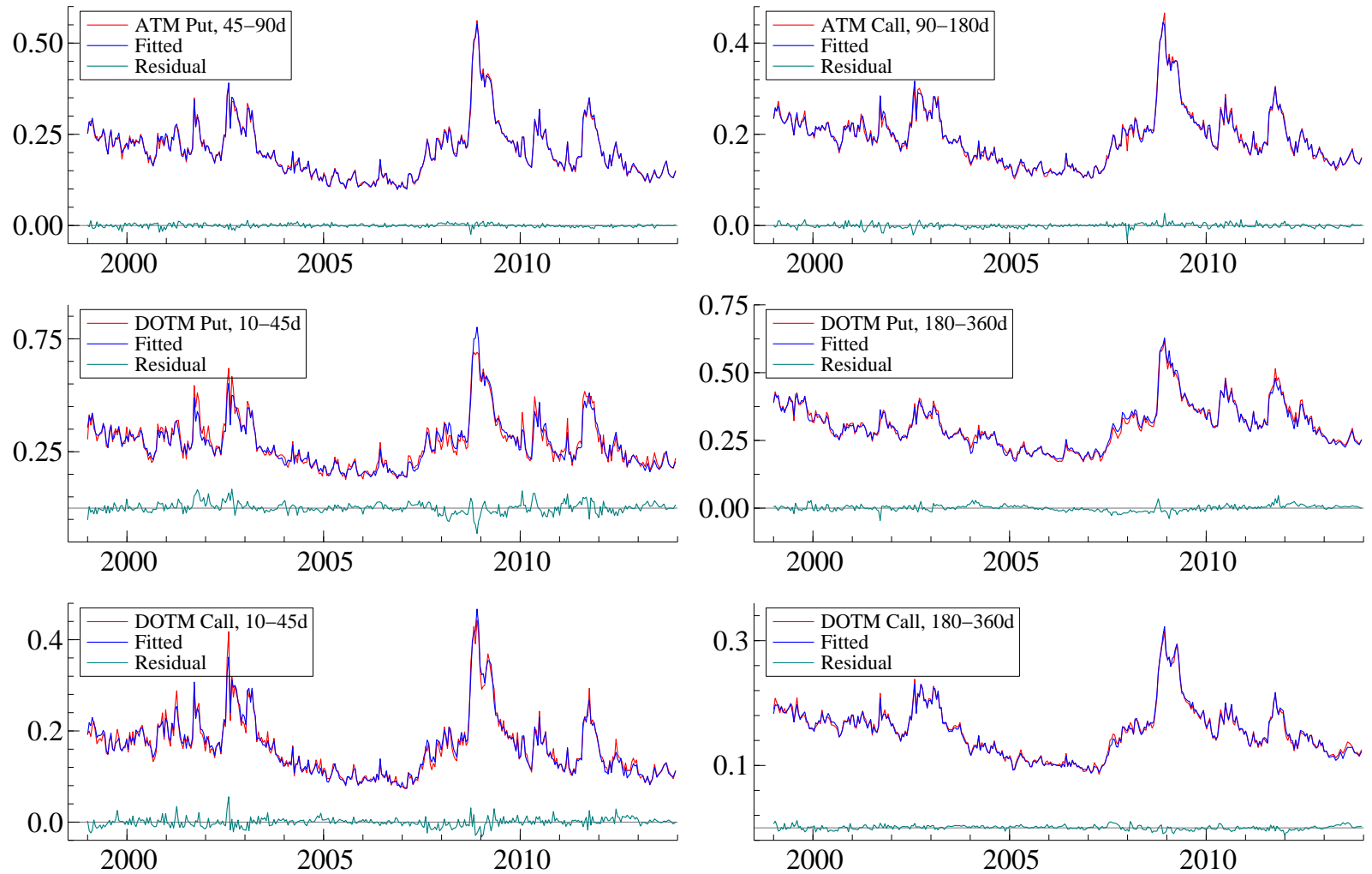
Out of the 24 groups in our data, we show a selection of six. The top two plots are fairly in the center of the surface, while the bottom four plots are on the corners. Overall the model fits the data fairly well for the points in the center of the surface. Fitting the corners appears to be more problematic, since the residuals do not look like merely white noise. In Figure A3, we provide similar figures for the restricted and spline-based factor models. The same conclusion is drawn for these models, though the problems in fitting the corners of the surface are much more serious for the restricted economic model.

\section{$5 \quad$ Robustness and Extensions}

Here we consider a number of robustness checks and extensions of our main modeling approach. First, Section 5.1 considers an alternative construction of the volatility surface based on moneyness measured by strike relative to spot price. Second, 5.2 examines some of the higher-dimensional restricted models that have been proposed in the literature. Third, Section 5.3 considers random walks for the factor dynamics. Finally, to examine to what extent the financial crisis dominates our results, 5.4 shows results when the analysis is done using log-transformed data or a sub-sample omitting the crisis.

\subsection{Alternative surface construction}

One of our main findings discussed in Section 4 is that $\Delta$ is a better measure of moneyness for building models of the volatility surface compared to the strike relative to the spot price. One possible explanation for this finding is that in fact it is spurious, since the construction of the surface is based on $\Delta$. To examine this possibility and the sensitivity of our results to the surface construction, we re-estimate all DFM specifications with the surface constructed using the strike relative to the spot price. Similar to before, we consider six moneyness groups, but they are now defined based on $K / S$ rather than $\Delta$. The ranges of the striketo-spot ratio that define the six groups are: $0<K / S<0.9$ (deep out-of-the-money put options), $0.9<K / S<0.95$ (out-of-the-money puts), $0.95<K / S<1$ (at-the-money puts), $1<K / S<1.05$ (at-the-money call options), $1.05<\Delta<1.1$ (out-of-the-money calls) and $K / S>1.1$ (deep out-of-the-money calls). We keep the definitions for the maturity groups the same, as given in Section 2.1. On each day we again find the option that is closest to 
the middle of each maturity-moneyness group, except for the deep out-of-the-money groups, where for moneyness we consider options that are closest to 0.85 and 1.15 for put and call options, respectively. ${ }^{13}$ Table A4 in the web appendix provides summary statistics for the obtained surface.

Table 4: Comparing Restricted Dynamic Factor Models - Surface Based on $K / S$ This table provides statistics concerning the fit of the three dynamic factor models, when estimated using the full sample of daily S\&P500 implied volatility surfaces over the period January 4, 1999 - August 30, 2013, when the surface is constructed based on the strike price relative to the spot price. The first model is the general dynamic factor model ('General DFM'), where only identification restrictions have been imposed. The second set of models are the restricted factor models based on the economic literature ('Restr DFM'). We estimate four variants, depending on whether the loading matrix is constant ('Const') or timevarying ('TV') and whether strike relative to stock price (' $K / S$ ') or $\Delta$ is used for moneyness. The third set of models are spline-based ('Spline DFM'). We consider the block dynamic factor model with knots on all places ('Block DFM'), a model with one spline for the maturity and one spline for the moneyness dimensions ('Mat and Mon Splines') and a model where there are separate splines for all maturity and moneyness groups ('Separate Splines'). For each model, we provide the log-likelihood value ('Loglik'), the number of parameters ('\#Pars') and the Akaike Information Criterion ('AIC'). For the restricted economic and spline models, we also provide a likelihood-ratio test ('LR-test') relative to the general dynamic factor model.

\begin{tabular}{llrrrrr}
\hline \multicolumn{6}{c}{ Comparison of Factor Models - Surface based on $K / S$} \\
\hline \multicolumn{2}{l}{ Model and Moneyness } & Const/TV & Loglik & LR-test & \#Pars & AIC \\
\hline General DFM & (only ident restr) & 288112.8 & & 102 & -576021.6 \\
\hline Restr DFM & $K / S$ & Const & 252946.3 & 70333.0 & 42 & -505808.6 \\
& & TV & 258878.7 & 58468.2 & 42 & -517673.4 \\
& $\Delta$ & Const & 255382.1 & 65461.4 & 42 & -510680.2 \\
& & TV & 262205.1 & 51815.4 & 42 & -524326.2 \\
\hline \multirow{2}{*}{ Spline DFM } & Block DFM & 260100.6 & 56024.4 & 50 & -520101.2 \\
& Mat and Mon Splines & 259325.6 & 57574.4 & 47 & -518557.2 \\
& Separate Splines & 279941.7 & 16342.2 & 66 & -559751.4 \\
\hline
\end{tabular}

Table 4 reports the estimation results based on this alternative volatility surface data. Focusing first on the restricted models, the table shows that even when the data are constructed based on $K / S$, using $\Delta$ in the model for the volatility surface still provides a better fit. An explanation of the robust finding that $\Delta$ should be preferred over the strike relative to the spot price is that the latter measure does not take into account that the likelihood of the option being in-the-money at expiration depends on the (current) volatility and remaining time-to-maturity, as Bollen and Whaley (2004) point out. Broadening our view to all models in the table, the ranking of the models remains unchanged relative to the baseline

\footnotetext{
${ }^{13}$ When using the middle of the deep out-of-the-money groups, the options are very deep out-of-the-money and fairly illiquid. This is less the case for $\Delta$, as this is a nonlinear measure. We also estimate all models by selecting the deep out-of-the-money groups in the middle and all results are qualitatively similar (such as the relative fit of the models), but the overall fit decreases.
} 
results in Table 2. Also for this alternative construction of the surface all restricted and spline-based models are rejected. The spline models still perform better than the restricted models, though the difference has become smaller and a model based on $\Delta$ with time-varying loadings outperforms two of the three spline models.

\section{$5.2 \quad$ Higher-dimensional models}

So far we have focused on 3-factor models. Dumas, Fleming, and Whaley (1998) consider models of up to six factors. In this section we consider these higher-order models to examine how they compare to the general dynamic factor model. We base our analysis on the model set-up from Section 3.2. We extend equation (2) with additional factors to match the higher-dimensional models. Specifically, to obtain a specification with four factors, we add the square of moneyness as additional factor. For the 5-factor case, we further add an interaction between moneyness and maturity and, finally, to arrive at the model with six factors we add the square of maturity. Similar to before when considering the restricted models, we study four variants depending on the type of the moneyness measure and whether the loading matrix is constant or time-varying.

Table 5 provides the output from this analysis. Obviously, the log-likelihood increases with the number of factors. The increase is not that large though, leading to the conclusion that a substantial number of factors is needed to fit the volatility surface well using these restricted models. In fact, out of all the higher-order models only the 6-factor time-varying loading models perform better than a 3-factor general dynamic factor model with constant loadings. For all dimensions, the time-varying loading matrix case outperforms the constant loading case. Also the conclusion that $\Delta$ is preferred over strike relative to stock price holds up irrespective of the number of factors.

\subsection{Alternative factor dynamics}

The results in Table 3 document a very strong persistence in the factor dynamics. We examine this aspect in more detail by considering two alternatives for the factor dynamics. As the persistence of the first factor is strongest in all model specifications, the first alternative considers a random walk for this factor while retaining an unrestricted first-order autore- 
Table 5: Comparing Higher-Order Restricted Dynamic Factor Models

This table provides statistics concerning the fit of the higher-dimensional restricted dynamic factor models, when estimated using the full sample of daily S\&P500 implied volatility surfaces over the period January 4, 1999 - August 30, 2013. The model with four factors explains the surface with a constant, moneyness, the square of moneyness and maturity. The model with five factors adds an interaction between moneyness and maturity, and the 6-factor model adds the square of maturity as additional factor. In all cases we estimate four variants, depending on whether the loading matrix is constant ('Const') or time-varying ('TV') and whether strike relative to stock price (' $K / S$ ') or $\Delta$ is used for moneyness. For each model, we provide the log-likelihood value ('Loglik'), the number of parameters ('\#Pars') and the Akaike Information Criterion ('AIC').

\begin{tabular}{lllrrr}
\hline \multicolumn{5}{c}{ Comparison of Restricted Factor Models } \\
\hline \#Factors & Moneyness & Const/TV & Loglik & \#Pars & AIC \\
\hline 4 & $K / S$ & Const & 260723.0 & 54 & -521338.0 \\
& \multirow{2}{*}{$\Delta$} & TV & 266423.5 & 54 & -532739.0 \\
& & Const & 278090.2 & 54 & -556072.4 \\
& TV & 287811.3 & 54 & -575514.6 \\
\hline $5 / S$ & Const & 284037.4 & 69 & -567936.8 \\
& \multirow{2}{*}{$\Delta$} & TV & 292112.6 & 69 & -584087.2 \\
& & Const & 284469.8 & 69 & -568801.6 \\
& \multirow{2}{*}{$K / S$} & TV & 296794.7 & 69 & -593451.4 \\
\hline \multirow{2}{*}{$\Delta$} & Const & 294499.2 & 87 & -588824.4 \\
& & TV & 305685.8 & 87 & -611197.6 \\
& & Const & 296505.8 & 87 & -592837.6 \\
& & TV & 311932.4 & 87 & -623690.8 \\
\hline
\end{tabular}

gressive specification for the second and third factors. The resulting VAR-coefficient model has a block-structure with 1 on the top-diagonal and a square block on the bottom-part with the coefficients for the second and third factor. As also the second and third factors seem persistent, we also run an alternative where we consider random walks for all three factors. In this case the VAR-coefficient matrix is simply an identity matrix. In these alternatives the intercept in the dynamics are estimated as $\mu$, rather than $(I-\Phi) \mu$ as in (1).

The alternatives are considered for all three main models that are reported in Table 3. In all cases the likelihood decreases for the alternative factor dynamics. LR-tests reject the imposed restrictions on the factor dynamics. Table A5 in the web appendix reports the factor dynamics for the regular case and the two alternatives mentioned above. In both of the alternatives the intercept is estimated at zero. When the level is modeled as a random walk the lower-block with the VAR-coefficients for the second and third factor remains unchanged. Also the covariance matrix is very similar. When all factors are modeled as random walks the covariance matrix is again very similar. These findings are similar for the restricted and spline-based models. Overall, the likelihood-ratio tests reject the imposed 
restrictions, but the similarity in findings when random walks are used for the factors hints that they are at least close to being non-stationary.

\subsection{Alternative sample period and log-transformation}

Figure 2 highlights the extreme volatility during the financial crisis in 2008-2009. We examine to what extent this special period determines our key findings. First, as alternative data we consider the log of implied volatility. Second, we consider a sub-sample from 1999 through 2007 and omit the crisis period altogether. As a first examination, Table A3 in the web appendix documents the percentage of explained variation for these two alternative data sets. In both cases, the first three factors combined explain close to $99 \%$ of the variation. The importance of the first factor decreases somewhat in the sub-sample analysis, but overall the factor structure is also very strong outside of the crisis period.

We also re-run the main models with these two alternative data sets (results unreported). The relative importance of the models does not change and also here $\Delta$ is preferred over strike relative to spot price as measure of moneyness. The persistence of the factors remains high, but decreases somewhat for the second and third factors. In the case of the logarithmic transformation the second diagonal element of $\Phi$ is equal to 0.97 and the third one equal to 0.95, while for the sub-sample analysis these are 0.95 and 0.85 , respectively.

\section{Conclusion}

This paper considers factor models for the implied volatility surface. Three main model types are considered. First, a general dynamic factor model where only identification restrictions are imposed. Second, restricted factor models that are inspired by linear models commonly used in the economic and financial literature. Third, spline-based models that offer a smooth alternative in-between the restrictive second model class and the heavily parameterized general dynamic factor model. The second and third model set-ups explicitly take into account that the data modeled is a three-dimensional surface.

We report three key findings. First, the economic and spline-based models are both rejected as restricted versions of the general dynamic factor model. The spline-based models are able to outperform the economic models, but even the best model is rejected by a 
likelihood-ratio test and not preferred based on information criteria. Second, the factors driving the surface are highly persistent. The VAR coefficient matrix has diagonal elements close to one. Third, for the restricted models $\Delta$ is preferred as a measure for moneyness over the strike relative to the spot price. Even if the surface is constructed based on the strike relative to the spot, the option $\Delta$ provides a better fit.

There are four main directions for further research. First, the spline-based models can be improved further by considering model selection in more detail. For example, in the context of term structure modeling Jungbacker, Koopman, and van der Wel (2014) offer testing procedures to select optimal 'smooth loadings'. A similar method can be used in the current setting. Second, due to the strong persistence it may be of interest to study nonstationary (factor) models for the volatility surface. Third, we study the factor structure using a balanced panel of 24 selected contracts each day based on pre-defined moneyness and maturity groups. An alternative is to consider the entire cross-section of individual option contracts. A fourth and final direction for further research is to study the forecasting performance of the various models. There is a recent literature finding supportive evidence that various existing models have forecasting power for the volatility surface, see, e.g., Konstantinidi, Skiadopoulos, and Tzagkaraki (2008), Chalamandaris and Tsekrekos (2010) and Bernales and Guidolin (2014). These studies consider a subset of our models and do not include the spline-based models that combine the flexibility of the general dynamic factor model with the economically plausible factor interpretation of the restricted models.

\section{References}

Barone-Adesi, G., R. F. Engle, and L. Mancini (2008). A GARCH option pricing model with filtered historical simulation. Rev. Financial Studies 21, 1223-1258.

Bedendo, M. and S. D. Hodges (2009). The dynamics of the volatility skew: A Kalman filter approach. J. Banking and Finance 33, 1156-1165.

Bernales, A. and M. Guidolin (2014). Can we forecast the implied volatility surface dynamics of equity options? Predictability and economic value tests. J. Banking and Finance 46, 326-342. 
Black, F. and M. Scholes (1973). The pricing of options and corporate liabilities. J. Political Economy 81, 637-654.

Bollen, N. P. and R. E. Whaley (2004). Does net buying pressure affect the shape of implied volatility functions? J. Finance 59, 711-753.

Chalamandaris, G. and A. E. Tsekrekos (2010). Predictable dynamics in implied volatility surfaces from OTC currency options. J. Banking and Finance 34, 1175-1188.

Christoffersen, P. F., M. Fournier, and K. Jacobs (2013). The factor structure in equity options. Working Paper.

Christoffersen, P. F., R. Goyenko, K. Jacobs, and M. Karoui (2012). Illiquidity premia in the equity options market. Working Paper.

Cont, R. and J. da Fonseca (2002). Dynamics of implied volatility surfaces. Quantitative Finance 2, 45-60.

Cox, J. C., S. A. Ross, and M. Rubinstein (1979). Option pricing: A simplified approach. J. Financial Economics 7, 229-263.

Dumas, B., J. Fleming, and R. E. Whaley (1998). Implied volatility functions: Empirical tests. J. Finance 53, 2059-2106.

Fengler, M. R., W. K. Härdle, and E. Mammen (2007). A semiparametric factor model for implied volatility surface dynamics. J. Financial Econometrics 5, 189-218.

Fengler, M. R., W. K. Härdle, and C. Villa (2003). The dynamics of implied volatilities: A common principal components approach. Rev. Derivatives Research 6, 179-202.

Geweke, J. and G. Zhou (1996). Measuring the pricing error of the arbitrage pricing theory. Rev. Financial Studies 9, 557-87.

Gonçalves, S. and M. Guidolin (2006). Predictable dynamics in the S\&P500 index options implied volatility surface. J. Business 79, 1591-1635.

Jungbacker, B. and S. J. Koopman (2014). Likelihood-based dynamic factor analysis for measurement and forecasting. Econometrics J., forthcoming.

Jungbacker, B., S. J. Koopman, and M. van der Wel (2014). Smooth dynamic factor analysis with application to the U.S. term structure of interest rates. J. Applied Econo- 
metrics 29, 65-90.

Konstantinidi, E., G. Skiadopoulos, and E. Tzagkaraki (2008). Can the evolution of implied volatility be forecasted? Evidence from European and US implied volatility indices. J. Banking and Finance 32, 2401-2411.

Mönch, E. and S. Ng (2011). A hierarchical factor analysis of U.S. housing market dynamics. Econometrics J. 14, C1-C24.

Park, B., E. Mammen, W. Härdle, and S. Borak (2009). Time series modelling with semiparametric factor dynamics. J. American Statistical Association 104, 284-98.

Poirier, D. J. (1976). The econometrics of structural change: With special emphasis on spline functions. Amsterdam: North-Holland.

Rubinstein, M. (1994). Implied binomial trees. J. Finance 49, 771-818.

Skiadopoulos, G., S. Hodges, and L. Clewlow (1999). The Dynamics of the S\&P500 Implied Volatility Surface. Rev. Derivatives Research 3, 263-282.

Stock, J. H. and M. W. Watson (2002). Macroeconomic forecasting using diffusion indexes. J. Business and Economic Statist. 20, 147-62. 
Appendix Tables and Figures 


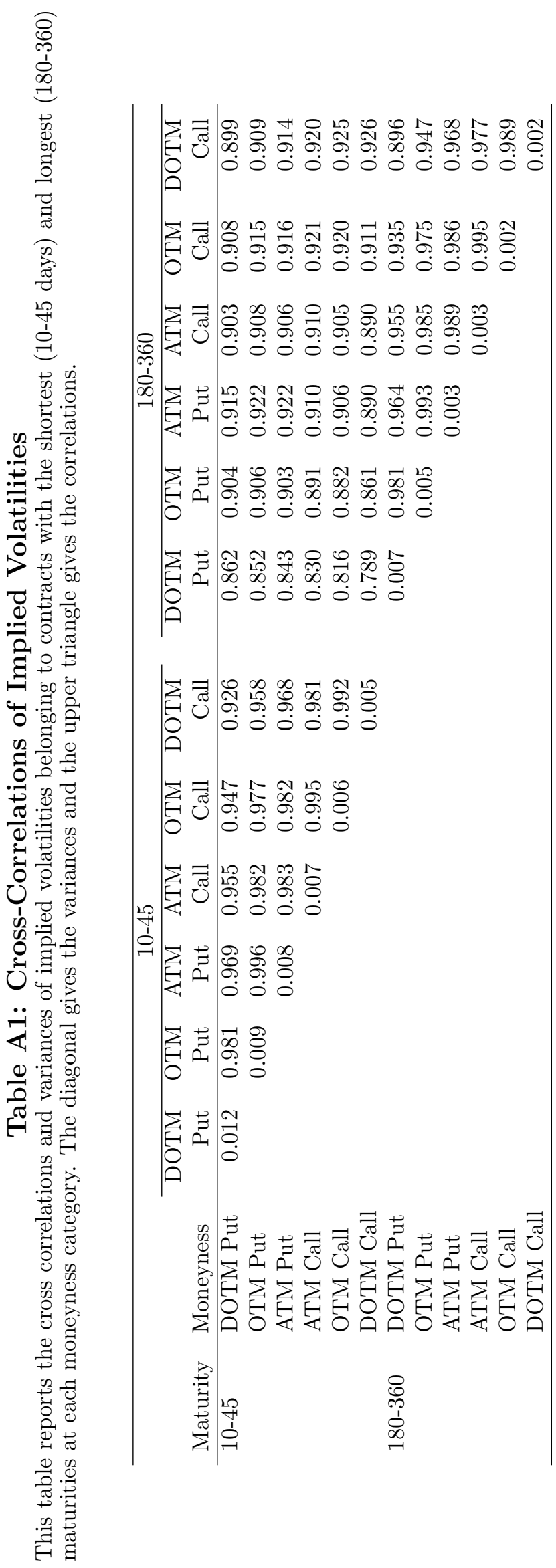




\section{Table A2: Persistence of the Volatility Surface}

This table provides (partial) autocorrelations for the volatility surface. Panel A presents the persistence of each moneyness category for the shortest (10-45 days) and longest (180-360) maturities. Panels B and C show the persistence for the slope of the volatility smile at different maturities and the slope of the term structure at different moneyness levels, respectively. The slope of the volatility smile in each of the four maturity groups is defined as the implied volatility of the deep out-of-the-money put minus the implied volatility of the deep out-of-the-money call. The slope of the volatility term structure for each of the six moneyness groups is defined as the implied volatility of the longest maturity minus the shortest maturity. (Partial) autocorrelations are given for lags 1, 2, 3, 5 and 20.

\begin{tabular}{|c|c|c|c|c|c|c|c|c|c|c|c|}
\hline \multicolumn{12}{|c|}{ Panel A: Implied Volatilities } \\
\hline \multirow[b]{2}{*}{ Moneyness } & \multirow[b]{2}{*}{ Maturity } & \multicolumn{5}{|c|}{$\mathrm{ACF}$} & \multicolumn{5}{|c|}{$\mathrm{PACF}$} \\
\hline & & 1 & 2 & 3 & 5 & 20 & 1 & 2 & 3 & 5 & 20 \\
\hline \multirow[t]{2}{*}{ DOTM Put } & $10-45$ & 0.975 & 0.955 & 0.941 & 0.919 & 0.803 & 0.975 & 0.112 & 0.106 & 0.045 & -0.014 \\
\hline & $180-360$ & 0.992 & 0.986 & 0.980 & 0.971 & 0.902 & 0.992 & 0.145 & 0.056 & 0.045 & -0.036 \\
\hline \multirow[t]{2}{*}{ OTM Put } & $10-45$ & 0.975 & 0.960 & 0.948 & 0.930 & 0.808 & 0.975 & 0.167 & 0.111 & 0.027 & -0.011 \\
\hline & $180-360$ & 0.991 & 0.985 & 0.980 & 0.970 & 0.900 & 0.991 & 0.142 & 0.073 & 0.050 & -0.022 \\
\hline \multirow[t]{2}{*}{ ATM Put } & $10-45$ & 0.974 & 0.958 & 0.947 & 0.929 & 0.812 & 0.974 & 0.183 & 0.131 & 0.048 & -0.033 \\
\hline & $180-360$ & 0.991 & 0.986 & 0.981 & 0.971 & 0.905 & 0.991 & 0.180 & 0.054 & 0.036 & 0.002 \\
\hline \multirow[t]{2}{*}{ ATM Call } & $10-45$ & 0.967 & 0.952 & 0.942 & 0.923 & 0.811 & 0.967 & 0.250 & 0.165 & 0.070 & -0.035 \\
\hline & $180-360$ & 0.991 & 0.986 & 0.981 & 0.972 & 0.915 & 0.991 & 0.234 & 0.082 & 0.044 & 0.037 \\
\hline \multirow[t]{2}{*}{ OTM Call } & $10-45$ & 0.972 & 0.957 & 0.944 & 0.925 & 0.818 & 0.972 & 0.219 & 0.101 & 0.085 & -0.024 \\
\hline & $180-360$ & 0.991 & 0.986 & 0.981 & 0.972 & 0.918 & 0.991 & 0.190 & 0.069 & 0.052 & -0.001 \\
\hline \multirow[t]{2}{*}{ DOTM Call } & $10-45$ & 0.971 & 0.955 & 0.942 & 0.922 & 0.825 & 0.971 & 0.205 & 0.096 & 0.093 & -0.016 \\
\hline & $180-360$ & 0.991 & 0.986 & 0.981 & 0.972 & 0.917 & 0.991 & 0.213 & 0.088 & 0.033 & -0.014 \\
\hline
\end{tabular}

\begin{tabular}{|c|c|c|c|c|c|c|c|c|c|c|}
\hline \multicolumn{11}{|c|}{ Panel B: Slope of Volatility Smile } \\
\hline \multirow[b]{2}{*}{ Maturity } & \multicolumn{5}{|c|}{$\mathrm{ACF}$} & \multicolumn{5}{|c|}{$\mathrm{PACF}$} \\
\hline & 1 & 2 & 3 & 5 & 20 & 1 & 2 & 3 & 5 & 20 \\
\hline $10-45$ & 0.930 & 0.882 & 0.846 & 0.799 & 0.655 & 0.930 & 0.130 & 0.085 & 0.098 & -0.012 \\
\hline $45-90$ & 0.971 & 0.953 & 0.937 & 0.912 & 0.774 & 0.971 & 0.189 & 0.065 & 0.036 & -0.018 \\
\hline $90-180$ & 0.978 & 0.968 & 0.959 & 0.943 & 0.829 & 0.978 & 0.255 & 0.090 & 0.054 & -0.008 \\
\hline $180-360$ & 0.984 & 0.976 & 0.970 & 0.958 & 0.874 & 0.984 & 0.252 & 0.098 & 0.058 & -0.011 \\
\hline
\end{tabular}

\begin{tabular}{|c|c|c|c|c|c|c|c|c|c|c|}
\hline \multicolumn{11}{|c|}{ Panel C: Slope of Volatility Term Structure } \\
\hline \multirow[b]{2}{*}{ Moneyness } & \multicolumn{5}{|c|}{$\mathrm{ACF}$} & \multicolumn{5}{|c|}{$\mathrm{PACF}$} \\
\hline & 1 & 2 & 3 & 5 & 20 & 1 & 2 & 3 & 5 & 20 \\
\hline DOTM Put & 0.934 & 0.889 & 0.861 & 0.821 & 0.648 & 0.934 & 0.137 & 0.131 & 0.065 & -0.007 \\
\hline OTM Put & 0.933 & 0.899 & 0.875 & 0.843 & 0.640 & 0.933 & 0.213 & 0.128 & 0.035 & -0.015 \\
\hline ATM Put & 0.933 & 0.895 & 0.873 & 0.839 & 0.631 & 0.933 & 0.193 & 0.157 & 0.046 & -0.043 \\
\hline ATM Call & 0.923 & 0.888 & 0.871 & 0.835 & 0.635 & 0.923 & 0.242 & 0.185 & 0.069 & -0.054 \\
\hline OTM Call & 0.932 & 0.897 & 0.869 & 0.831 & 0.632 & 0.932 & 0.219 & 0.091 & 0.088 & -0.022 \\
\hline DOTM Call & 0.925 & 0.886 & 0.853 & 0.811 & 0.631 & 0.925 & 0.209 & 0.085 & 0.092 & -0.014 \\
\hline
\end{tabular}


Table A3: Principal Component Analysis

This table shows results of a principal component analysis on the panel of 24 implied volatilities that represent the volatility surface at each point in time. Panel A reports the percentage of variation explained by each individual principal component series (first column) and the cumulative percentage (second column). Panel B reports (partial) autocorrelations of the first three principal components for lags 1, 2, 3, 5 and 20. In addition, Panel A reports the explained variation for two alternative data sets, where either the log of implied volatility is considered or only the 1999-2007 sub-sample.

\begin{tabular}{|c|c|c|c|c|c|c|}
\hline \multicolumn{7}{|c|}{ Panel A: Expl. Variation } \\
\hline & \multicolumn{2}{|c|}{ Regular Data } & \multicolumn{2}{|c|}{ Log-transformation } & \multicolumn{2}{|c|}{ 1999-2007 } \\
\hline & $\%$ & Cum. \% & $\%$ & Cum. \% & $\%$ & Cum. \% \\
\hline $\mathrm{PC} 1$ & 95.19 & 95.19 & 96.00 & 96.00 & 94.72 & 94.72 \\
\hline PC 2 & 2.69 & 97.88 & 2.08 & 98.08 & 2.80 & 97.52 \\
\hline $\mathrm{PC} 3$ & 1.09 & 98.97 & 1.03 & 99.11 & 1.29 & 98.81 \\
\hline PC 4 & 0.29 & 99.26 & 0.19 & 99.30 & 0.30 & 99.11 \\
\hline PC 5 & 0.22 & 99.49 & 0.17 & 99.48 & 0.24 & 99.35 \\
\hline PC 6 & 0.15 & 99.63 & 0.14 & 99.61 & 0.15 & 99.50 \\
\hline $\mathrm{PC} 7$ & 0.09 & 99.72 & 0.13 & 99.74 & 0.14 & 99.63 \\
\hline PC 8 & 0.07 & 99.79 & 0.05 & 99.80 & 0.10 & 99.73 \\
\hline PC 9 & 0.04 & 99.84 & 0.03 & 99.83 & 0.05 & 99.78 \\
\hline PC 10 & 0.03 & 99.87 & 0.03 & 99.86 & 0.04 & 99.82 \\
\hline
\end{tabular}

\begin{tabular}{|c|c|c|c|c|c|c|c|c|c|c|}
\hline \multicolumn{11}{|c|}{ Panel B: Persistence of Principal Components (Regular Data) } \\
\hline & \multicolumn{5}{|c|}{$\mathrm{ACF}$} & \multicolumn{5}{|c|}{ PACF } \\
\hline & 1 & 2 & 3 & 5 & 20 & 1 & 2 & 3 & 5 & 20 \\
\hline PC1 & 0.988 & 0.978 & 0.970 & 0.957 & 0.868 & 0.988 & 0.108 & 0.080 & 0.046 & -0.018 \\
\hline $\mathrm{PC} 2$ & 0.958 & 0.934 & 0.917 & 0.891 & 0.747 & 0.958 & 0.189 & 0.132 & 0.046 & 0.002 \\
\hline PC3 & 0.879 & 0.826 & 0.787 & 0.729 & 0.508 & 0.879 & 0.236 & 0.118 & 0.085 & -0.040 \\
\hline
\end{tabular}


Table A4: Summary Statistics - Surface Construction Based on $K / S$

This table shows summary statistics for the option data when the surface is constructed on the strike price relative to the stock price. The table provides the mean and standard deviation ('Sd') over time for the mid-quote (in dollars), implied volatility ('Impl Vol'), option $\Delta$, maturity (in days), and strike relative to stock price $(\mathrm{K} / \mathrm{S})$. We show these numbers across four maturity groups and six moneyness groups. The maturity groups are 10-45, 45-90, 90-180 and 180-360 days. The moneyness groups are $0<K / S<0.9$ (deep out-of-the-money put options, DOTM Put), $0.9<K / S<0.95$ (out-of-the-money puts, OTM Put), $0.95<K / S<1$ (at-the-money puts, ATM Put), $1<K / S<1.05$ (at-the-money call options, ATM Call), $1.05<\Delta<1.1$ (out-of-the-money calls, OTM Call) and $K / S>1.1$ (deep out-of-the-money calls, DOTM Call). On each day we find the option that is closest to the middle of each maturity-moneyness group, except for the DOTM groups, where for moneyness we consider options that are closest to 0.85 and 1.15 for put and call options, respectively. The numbers in the table represent averages over time for the selected contracts in each group.

\begin{tabular}{|c|c|c|c|c|c|c|c|c|c|}
\hline \multicolumn{10}{|c|}{ Summary Statistics } \\
\hline & & \multicolumn{2}{|c|}{ 10-45 days } & \multicolumn{2}{|c|}{ 45-90 days } & \multicolumn{2}{|c|}{ 90-180 days } & \multicolumn{2}{|c|}{$180-360$ days } \\
\hline & & Mean & Sd & Mean & $\mathrm{Sd}$ & Mean & $\mathrm{Sd}$ & Mean & $\mathrm{Sd}$ \\
\hline \multirow[t]{5}{*}{ DOTM Put } & Mid-Quote & 1.82 & 3.6 & 6.44 & 5.85 & 14.7 & 9.38 & 31.1 & 15 \\
\hline & Impl Vol & 0.344 & 0.0798 & 0.29 & 0.0739 & 0.271 & 0.0675 & 0.258 & 0.0599 \\
\hline & $\Delta$ & -0.0321 & 0.0323 & -0.079 & 0.0437 & -0.127 & 0.0488 & -0.18 & 0.0476 \\
\hline & Maturity & 27.5 & 8.67 & 67.4 & 8.55 & 133 & 23.1 & 270 & 24.7 \\
\hline & $\mathrm{K} / \mathrm{S}$ & 0.85 & 0.00513 & 0.85 & 0.00682 & 0.85 & 0.0108 & 0.85 & 0.0112 \\
\hline \multirow[t]{5}{*}{ OTM Put } & Mid-Quote & 5.57 & 5.8 & 15.3 & 9.16 & 28.5 & 13.1 & 50.4 & 18.8 \\
\hline & Impl Vol & 0.265 & 0.0824 & 0.245 & 0.0753 & 0.238 & 0.0674 & 0.234 & 0.0594 \\
\hline & $\Delta$ & -0.111 & 0.0607 & -0.192 & 0.0576 & -0.246 & 0.0515 & -0.287 & 0.0461 \\
\hline & Maturity & 27.4 & 8.34 & 67.3 & 8.66 & 133 & 23.8 & 270 & 26.2 \\
\hline & $\mathrm{K} / \mathrm{S}$ & 0.925 & 0.00359 & 0.925 & 0.00494 & 0.925 & 0.00689 & 0.925 & 0.00732 \\
\hline \multirow[t]{5}{*}{ ATM Put } & Mid-Quote & 14.2 & 8.57 & 28.4 & 11.4 & 44.9 & 15.1 & 69.1 & 21 \\
\hline & Impl Vol & 0.219 & 0.085 & 0.215 & 0.0756 & 0.216 & 0.0674 & 0.218 & 0.0591 \\
\hline & $\Delta$ & -0.293 & 0.0623 & -0.354 & 0.0426 & -0.377 & 0.0406 & -0.384 & 0.0431 \\
\hline & Maturity & 27.4 & 8.25 & 67.3 & 8.6 & 133 & 23 & 269 & 25.7 \\
\hline & $\mathrm{K} / \mathrm{S}$ & 0.975 & 0.00279 & 0.975 & 0.00446 & 0.975 & 0.00729 & 0.975 & 0.00724 \\
\hline \multirow[t]{5}{*}{ ATM Call } & Mid-Quote & 11.2 & 8.45 & 25.8 & 12.3 & 44.1 & 17.2 & 73 & 24 \\
\hline & Impl Vol & 0.182 & 0.0838 & 0.188 & 0.0734 & 0.194 & 0.0648 & 0.201 & 0.0565 \\
\hline & $\Delta$ & 0.283 & 0.0889 & 0.386 & 0.0576 & 0.441 & 0.0503 & 0.487 & 0.0493 \\
\hline & Maturity & 27.3 & 8.21 & 67.4 & 8.49 & 134 & 22.8 & 269 & 25.2 \\
\hline & $\mathrm{K} / \mathrm{S}$ & 1.03 & 0.00274 & 1.02 & 0.00441 & 1.03 & 0.0063 & 1.03 & 0.00713 \\
\hline \multirow[t]{5}{*}{ OTM Call } & Mid-Quote & 2.18 & 3.96 & 8.55 & 8.26 & 21.2 & 14 & 46 & 21.3 \\
\hline & Impl Vol & 0.177 & 0.0707 & 0.169 & 0.07 & 0.178 & 0.0639 & 0.188 & 0.056 \\
\hline & $\Delta$ & 0.0629 & 0.0661 & 0.157 & 0.0902 & 0.258 & 0.0864 & 0.36 & 0.068 \\
\hline & Maturity & 27.3 & 8.21 & 67.4 & 8.5 & 134 & 22.9 & 269 & 25 \\
\hline & $\mathrm{K} / \mathrm{S}$ & 1.07 & 0.00435 & 1.07 & 0.00511 & 1.07 & 0.00664 & 1.07 & 0.00767 \\
\hline \multirow[t]{5}{*}{ DOTM Call } & Mid-Quote & 0.41 & 1.32 & 1.66 & 3.24 & 6.15 & 7.17 & 20.6 & 15.1 \\
\hline & Impl Vol & 0.22 & 0.0675 & 0.169 & 0.0549 & 0.163 & 0.0574 & 0.172 & 0.0542 \\
\hline & $\Delta$ & 0.0129 & 0.0218 & 0.0366 & 0.0441 & 0.0914 & 0.0705 & 0.195 & 0.0852 \\
\hline & Maturity & 29.2 & 10.7 & 67.2 & 10.4 & 134 & 23 & 270 & 25.3 \\
\hline & $\mathrm{K} / \mathrm{S}$ & 1.14 & 0.0191 & 1.15 & 0.0143 & 1.15 & 0.0126 & 1.15 & 0.0105 \\
\hline
\end{tabular}




\section{Table A5: Alternative Factor Dynamics Specifications}

This table provides estimates of the factor dynamics for general dynamic factor model under three alternative specifications. We show the coefficients for the general dynamic factor model where the factor dynamics are estimated as usual (Panel A), when the level factor is restricted to be a unit root (Panel B), and when all factors are unit roots (Panel C). For all three models, we plot the intercept $\mu$, VAR coefficient matrix $\Phi$ and the innovation variance $\Sigma_{\eta}$.

\begin{tabular}{rrrrrrrrr}
\hline \multicolumn{8}{c}{ Panel A: Free Factor Dynamics General DFM } \\
\cline { 3 - 5 } \cline { 7 - 9 } & & \multicolumn{4}{c}{$\Phi$} & & \multicolumn{3}{c}{$\Sigma_{\eta}\left(\times 10^{-4}\right)$} \\
\hline$f_{1, t}$ & 0.196 & 0.995 & 0.028 & -0.005 & & 1.059 & -0.453 & 0.051 \\
$f_{2, t}$ & 0.003 & -0.002 & 0.971 & 0.056 & & -0.453 & 0.212 & -0.022 \\
$f_{3, t}$ & 0.008 & 0.001 & 0.001 & 0.967 & & 0.051 & -0.022 & 0.005 \\
\hline
\end{tabular}

\begin{tabular}{rrrrrrrrr}
\hline \multicolumn{3}{c}{ Panel B: Restricted Factor Dynamics DFM - Level Random Walk } \\
\hline & \multicolumn{4}{c}{$\Phi$} & \multicolumn{2}{c}{$\Phi$} & & \multicolumn{3}{c}{$\Sigma_{\eta}\left(\times 10^{-4}\right)$} \\
\cline { 3 - 4 } \cline { 7 - 9 } & & $l_{t-1}$ & $c_{t-1}$ & $s_{t-1}$ & & $l_{t}$ & $c_{t}$ & $s_{t}$ \\
\hline$f_{1, t}$ & 0.000 & 1 & 0 & 0 & & 1.064 & -0.454 & 0.051 \\
$f_{2, t}$ & 0.000 & 0 & 0.989 & -0.002 & & -0.454 & 0.211 & -0.022 \\
$f_{3, t}$ & 0.000 & 0 & -0.002 & 0.985 & & 0.051 & -0.022 & 0.005 \\
\hline
\end{tabular}

\begin{tabular}{rrrrrrrrr}
\hline \multicolumn{3}{c}{ Panel C: Restricted Factor Dynamics DFM - All Random Walks } \\
\hline & & \multicolumn{3}{c}{$\Phi$} & & \multicolumn{3}{c}{$\Sigma_{\eta}\left(\times 10^{-4}\right)$} \\
\cline { 3 - 4 } \cline { 7 - 9 } & $f_{1, t-1}$ & $f_{2, t-1}$ & $f_{3, t-1}$ & & $f_{1, t}$ & $f_{2, t}$ & $f_{3, t}$ \\
\hline$f_{1, t}$ & 0.000 & 1 & 0 & 0 & & 1.064 & -0.454 & 0.051 \\
$f_{2, t}$ & 0.000 & 0 & 1 & 0 & & -0.454 & 0.212 & -0.022 \\
$f_{3, t}$ & 0.000 & 0 & 0 & 1 & & 0.051 & -0.022 & 0.005 \\
\hline
\end{tabular}


Figure A1: Principal Components of Volatility Surface

This figure shows the first three principal components of the volatility surface. We run a principal component analysis on the panel of 24 implied volatilities that represent the volatility surface at each point in time. The red line represents the first principal component, the blue line the second principal component and the red line the third principal component.

First three Principal Components

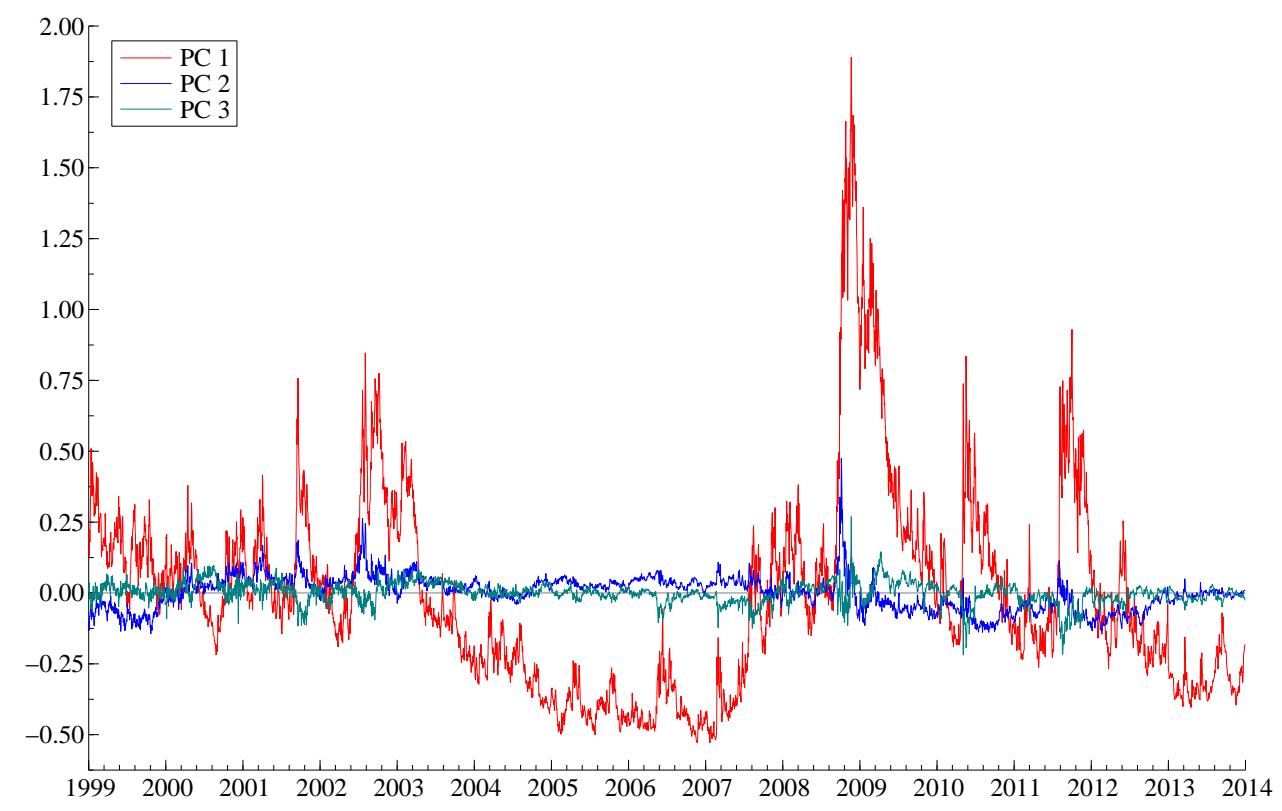




\section{Figure A2: Estimated Factors - Rotated to Restricted Factors}

This figure shows rotated dynamics factors for the factor models we consider. By regressing one factor loading matrix on another factor loading matrix we bring the loading matrices and the models closer together and obtain rotated factors. In this figure we show the resulting rotated factors of the general dynamic factor model, which are brought close to the restricted dynamic factor model with a constant loading matrix where moneyness is measured with $\Delta$ (Panel $\mathrm{A}$ ), and close to the spline dynamic factor model where there is a separate spline for each moneyness and maturity group (Panel B). The red line represents the first factor, the blue line the second factor and the red line the third factor. The solid lines denote the rotated factors, while the dotted lines denote the factors of the restricted and spline models in Panels A and B, respectively.

(A) General Dynamic Factor Model rotated to Restricted Economic Factors

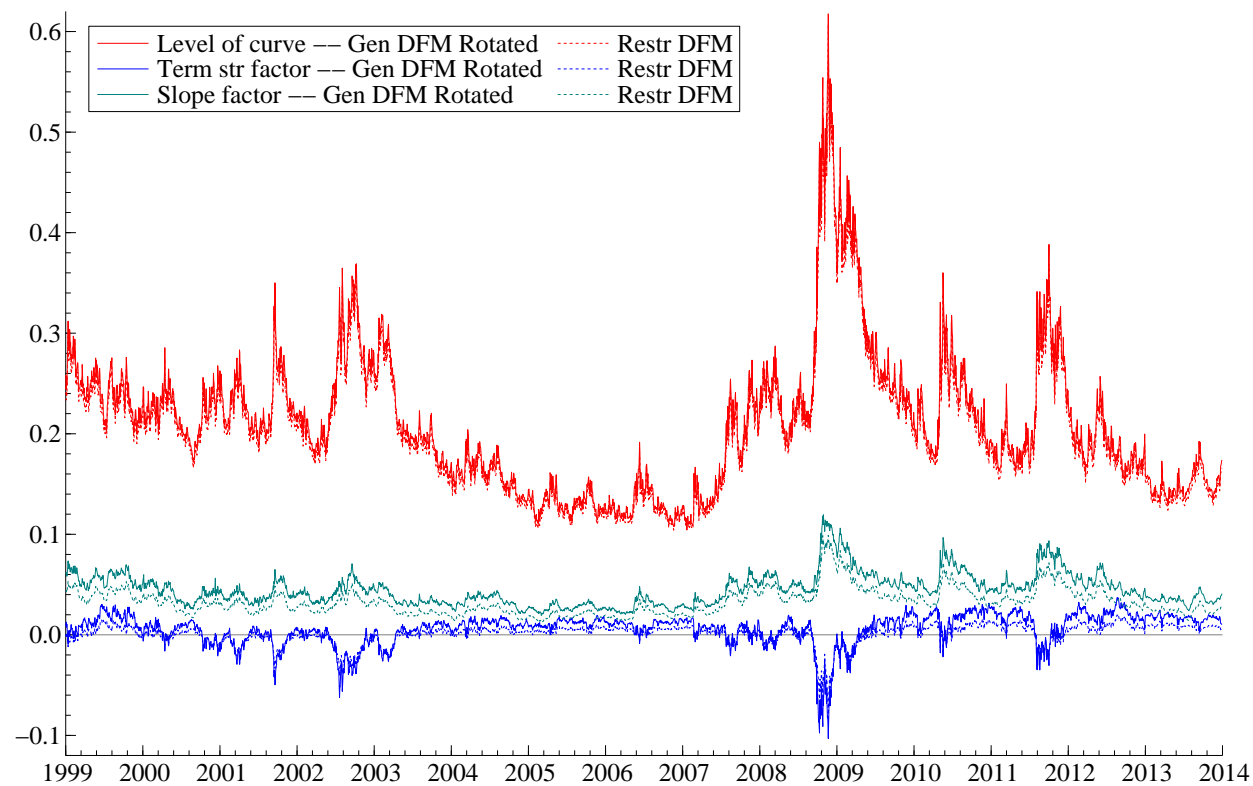

(B) General Dynamic Factor Model rotated to Spline Factors

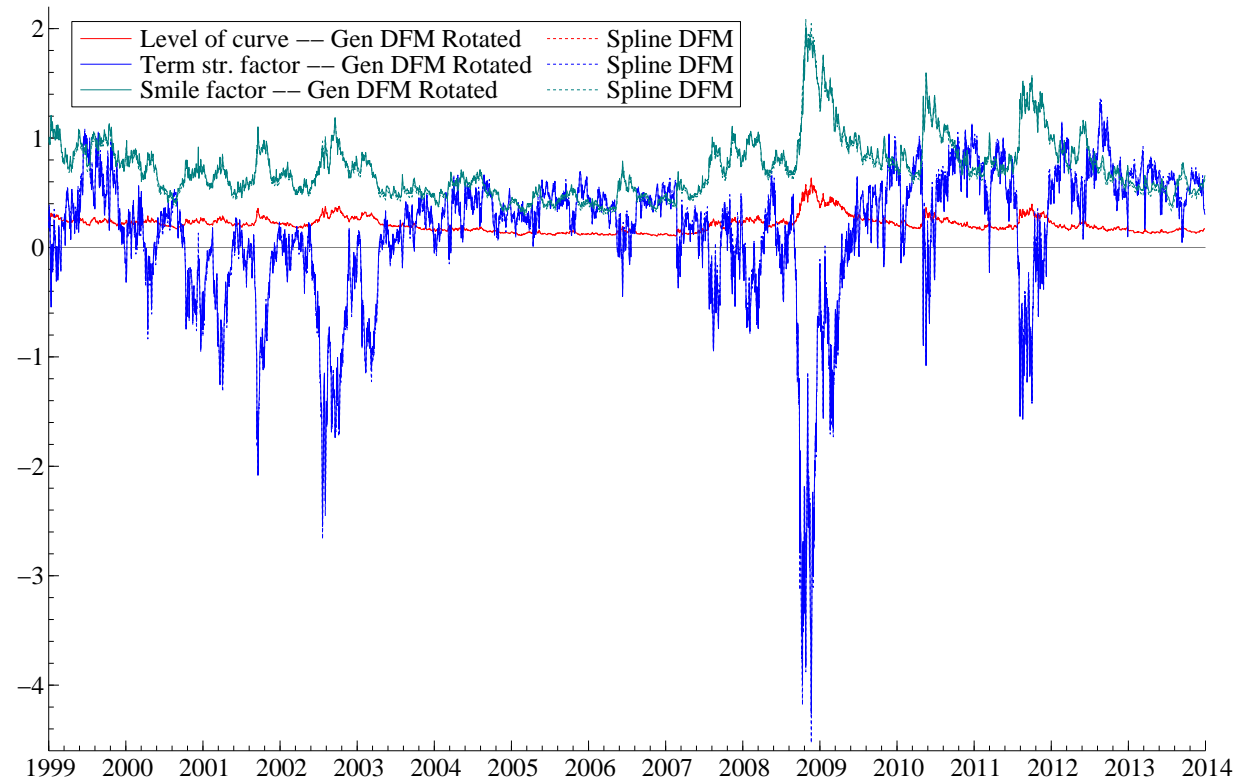


Figure A3: Fit

This figure documents the fit of the volatility surface based on two dynamic factor models. The models we consider are the restricted dynamic factor model with a constant loading matrix where moneyness is measured with $\Delta$ (Panel A), and the spline dynamic factor model where there is a separate spline for each moneyness and maturity group (Panel B). We show the smoothed fitted values, which are obtained by pre-multiplying the smoothed factors with the factor loading matrix. In the figure the red line is the actual implied volatility, the blue line the fitted and the green line the residual. We show six different maturitymoneyness combinations: the top plots depict two points in the middle of the surface and the bottom plots four outer points on the surface.

\section{(A) Restricted Economic Dynamic Factor Model}
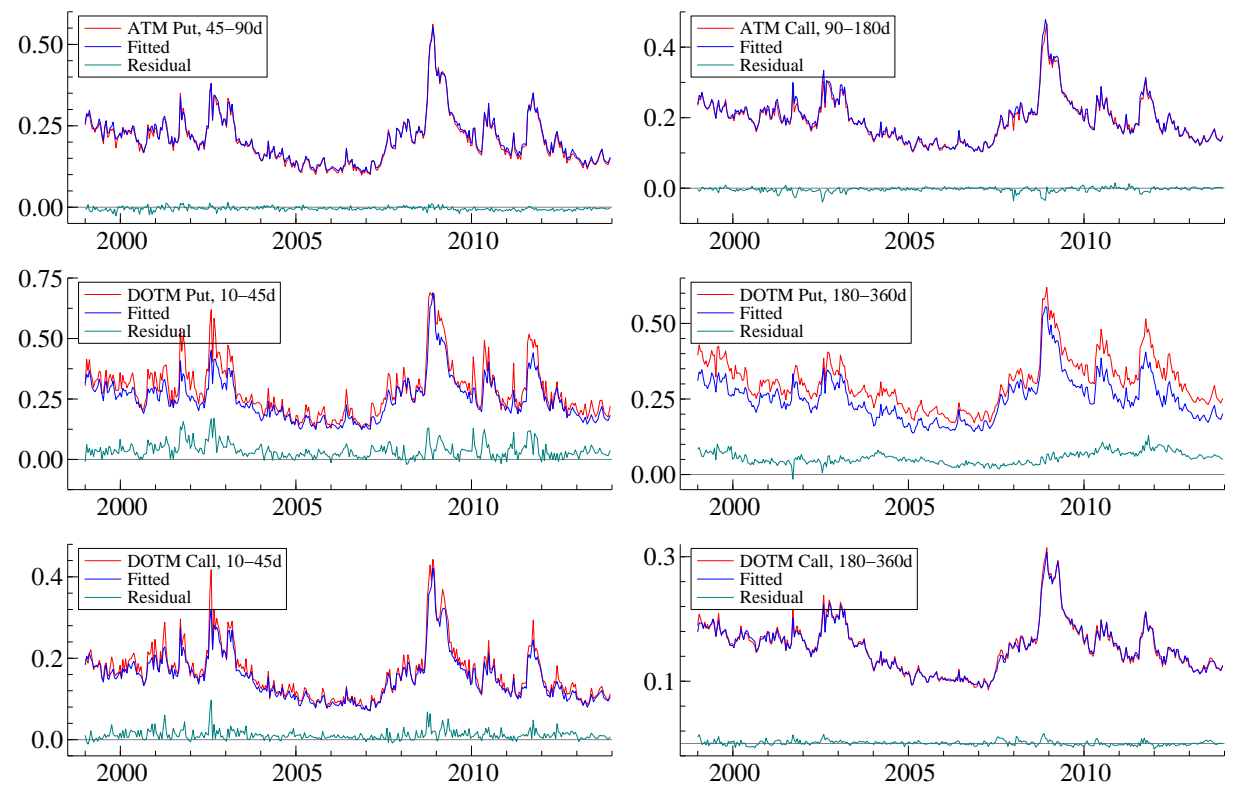

(B) Spline Dynamic Factor Model
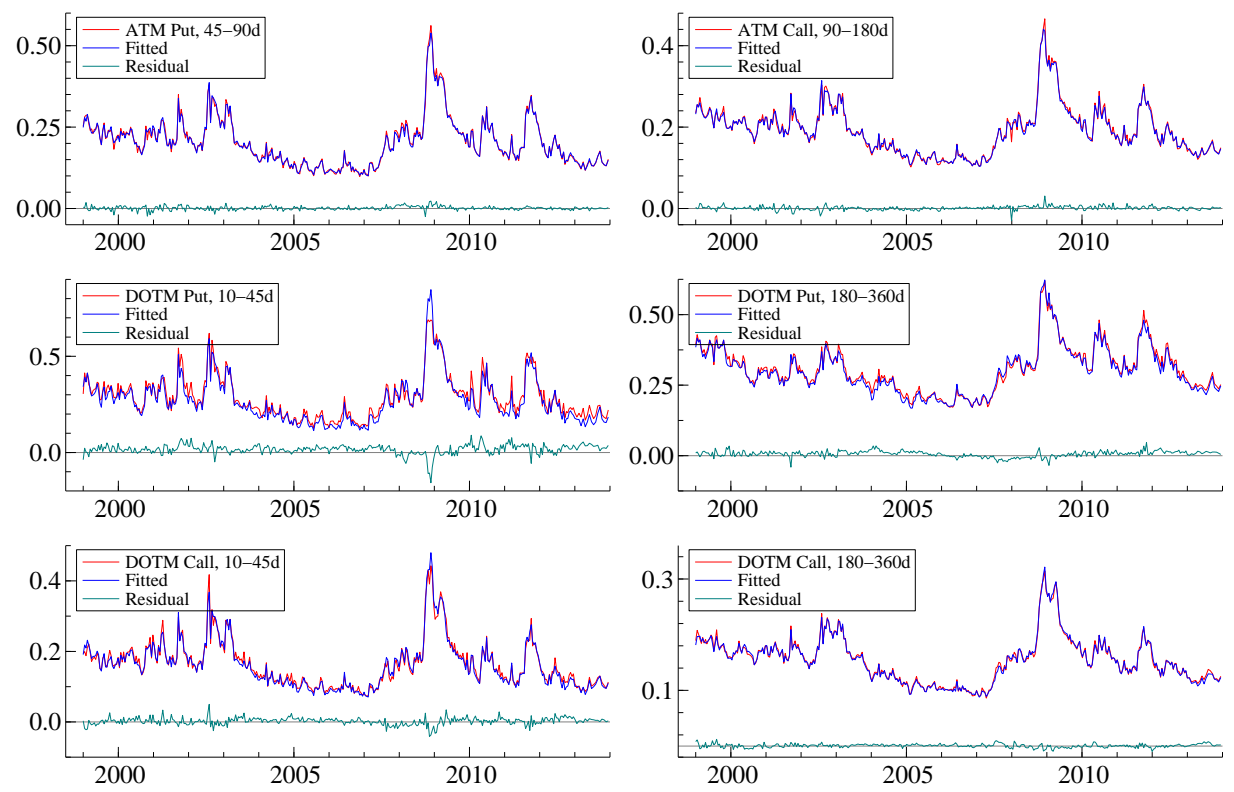
2014-54: Claudio Heinrich and Mark Podolskij: On spectral distribution of high dimensional covariation matrices

2014-55: $\quad$ Gustavo Fruet Dias and Fotis Papailias: Forecasting Long Memory Series Subject to Structural Change: A Two-Stage Approach

2014-56: Torben G. Andersen, Nicola Fusari and Viktor Todorov: The Risk Premia Embedded in Index Options

2014-57: $\quad$ Eduardo Rossi and Paolo Santucci de Magistris: Indirect inference with time series observed with error

2014-58: $\quad$ Anders Bredahl Kock and Haihan Tang: Inference in High-dimensional Dynamic Panel Data Models

2015-01 Tom Engsted, Simon J. Hviid and Thomas Q. Pedersen: Explosive bubbles in house prices? Evidence from the OECD countries

2015-02: $\quad$ Tim Bollerslev, Andrew J. Patton and Wenjing Wang: Daily House Price Indices: Construction, Modeling, and Longer-Run Predictions

2015-03: Christian M. Hafner, Sebastien Laurent and Francesco Violante: Weak diffusion limits of dynamic conditional correlation models

2015-04: $\quad$ Maria Eugenia Sanin, Maria Mansanet-Bataller and Francesco Violante: Understanding volatility dynamics in the EU-ETS market

2015-05: $\quad$ Peter Christoffersen and Xuhui (Nick) Pan: Equity Portfolio Management Using Option Price Information

2015-06: Peter Christoffersen and Xuhui (Nick) Pan: Oil Volatility Risk and Expected Stock Returns

2015-07: $\quad$ Peter Christoffersen, Bruno Feunou and Yoontae Jeon: Option Valuation with Observable Volatility and Jump Dynamics

2015-08: $\quad$ Alfonso Irarrazabal and Juan Carlos Parra-Alvarez: Time-varying disaster risk models: An empirical assessment of the Rietz-Barro hypothesis

2015-09: Daniela Osterrieder, Daniel Ventosa-Santaulària and Eduardo Vera-Valdés: Unbalanced Regressions and the Predictive Equation

2015-10: $\quad$ Laurent Callot, Mehmet Caner, Anders Bredahl Kock and Juan Andres Riquelme: Sharp Threshold Detection Based on Sup-norm Error rates in Highdimensional Models

2015-11: $\quad$ Arianna Agosto, Giuseppe Cavaliere, Dennis Kristensen and Anders Rahbek: Modeling corporate defaults: Poisson autoregressions with exogenous covariates (PARX)

2015-12: $\quad$ Tommaso Proietti, Martyna Marczak and Gianluigi Mazzi: EuroMInd-D: A Density Estimate of Monthly Gross Domestic Product for the Euro Area

2015-13: $\quad$ Michel van der Wel, Sait R. Ozturk and Dick van Dijk: Dynamic Factor Models for the Volatility Surface 